\title{
Stroke-related alterations in inter-areal communication
}

\author{
Michele Allegra $^{\mathrm{a}, *}$, Chiara Favaretto ${ }^{\mathrm{b}, \mathrm{c}}$, Nicholas Metcalf ${ }^{\mathrm{d}}$, Maurizio Corbetta ${ }^{\mathrm{b}, \mathrm{c}, \mathrm{d}, 1}$, \\ Andrea Brovelli ${ }^{a, 1}$ \\ ${ }^{a}$ Institut de Neurosciences de la Timone UMR 7289, Aix Marseille Université, CNRS, Marseille 13005, France \\ ${ }^{\mathrm{b}}$ Department of Neuroscience, Neurological Clinic, University of Padua, Padua, Italy \\ ${ }^{\mathrm{c}}$ Padova Neuroscience Center, University of Padua, Padua, Italy \\ d Department of Neurology, Radiology, and Neuroscience, Washington University in St. Louis, St. Louis, MO, United States
}

\section{A R T I C L E I N F O}

\section{Keywords:}

Stroke

Granger causality

Resting state fMRI

\begin{abstract}
A B S T R A C T
Beyond causing local ischemia and cell damage at the site of injury, stroke strongly affects long-range anatomical connections, perturbing the functional organization of brain networks. Several studies reported functional connectivity abnormalities parallelling both behavioral deficits and functional recovery across different cognitive domains. FC alterations suggest that long-range communication in the brain is altered after stroke. However, standard FC analyses cannot reveal the directionality and time scale of inter-areal information transfer. We used resting-state fMRI and covariance-based Granger causality analysis to quantify network-level information transfer and its alteration in stroke. Two main large-scale anomalies were observed in stroke patients. First, interhemispheric information transfer was significantly decreased with respect to healthy controls. Second, stroke caused inter-hemispheric asymmetries, as information transfer within the affected hemisphere and from the affected to the intact hemisphere was significantly reduced. Both anomalies were more prominent in resting-state networks related to attention and language, and they correlated with impaired performance in several behavioral domains. Overall, our findings support the hypothesis that stroke provokes asymmetries between the affected and spared hemisphere, with different functional consequences depending on which hemisphere is lesioned.
\end{abstract}

\section{Introduction}

Spontaneous brain activity is intrinsically organized into large-scale networks of correlated activity (Bullmore and Sporns, 2009; Damoiseaux et al., 2006; Fox et al., 2005), also known as resting-state networks (RSNs). The functional organization of RSNs is altered in stroke (Corbetta et al., 2018; Corbetta et al., 2015). In fact, local ischemia, which damages cells and neural connections at the site of injury, primarily affects subcortical regions and white matter, thus altering longrange functional connectivity (FC) between cortical areas. Two types of large-scale FC alterations affect RSNs (Siegel et al., 2016a): i) a decrease of within-network interhemispheric FC (Carter et al., 2010; Golestani et al., 2013; He et al., 2007; New et al., 2015; Park et al., 2011; Ramsey et al., 2016; Siegel et al., 2016a; Tang et al., 2016); ii) an increase of between-network intra-hemispheric FC (Baldassarre et al.,
2014; Eldaief et al., 2017; Ramsey et al., 2016; Siegel et al., 2016a). As a consequence, within-RSN connections are weakened, while betweenRSN connections are strengthened, which translates into an overall decrease of network modularity (Gratton et al., 2012). The presence of such common network-level perturbations explains why lesions in different locations in the brain produce remarkably similar behavioral deficits in different patients (Corbetta et al., 2018).

FC alterations suggest that behavioral deficits are due to the perturbation of inter-areal information flow. However, FC analyses cannot reveal the directionality or time scale of the information flow, leaving several questions open: i) is the stroke-related decrease of interhemispheric FC associated with a symmetric or asymmetric decrease in information flow between the damaged and non-damaged hemisphere? ii) is the increase of between-network intra-hemispheric FC paralleled by a change in intra-hemispheric information flow? iii) to

\footnotetext{
Abbreviations: fMRI, functional magnetic resonance imaging; FC, functional connectivity; RSNs, resting-state networks; GC, Granger causality; IC, instantaneous (Granger) causality; DC, directed (Granger) causality; UFC, undirected functional connectivity; LH/RH, left/right hemisphere.

* Corresponding author at: Institut de Neurosciences de la Timone (INT), UMR 7289 CNRS, Aix Marseille University, Campus de Santé Timone, 27 Bd. Jean Moulin, Marseille 13385, France.

E-mail addresses: michele.allegra@univ-amu.fr (M. Allegra), andrea.brovelli@univ-amu.fr (A. Brovelli).

1 Co-senior author.
} 
which extent do changes in network-level information flows predict cognitive deficits? To address these questions, we performed covariance-based Granger Causality (GC) analyses (Brovelli et al., 2015) of resting-state fMRI data collected from stroke patients in the sub-acute phase (two weeks after stroke onset). Data were provided by the Washington university stroke database (Corbetta et al., 2015), and included structural lesions, resting-state fMRI, and neuropsychological scores for a large cohort of first-time stroke patients and age-matched control subjects. Analyses revealed that inter-hemispheric information transfer was significantly decreased in stroke patients with respect to healthy controls. In addition, pronounced inter-hemispheric imbalances in information transfer were observed in patients. Both anomalies were more prominent in resting-state networks related to attention and language, and they paralleled deficits in several behavioral domains.

\section{Materials \& methods}

\subsection{Brain imaging and behavioral measurements}

Details about participants, neuroimaging data acquisition and preprocessing, and brain lesion identification can be found in previous publications on the same data set (Corbetta et al., 2015; Siegel et al., 2016b). Therefore, here we report only key information allowing for a self-contained reading of the paper.

Subject Enrollment and Retention. Participants $(\mathrm{n}=172)$ were prospectively recruited. First-time stroke patients with clinical evidence of motor, language, attention, visual, or memory deficits based on neurological examination were included. One hundred and thirty-two patients met all inclusion criteria (for details see Corbetta et al., 2015) and completed the entire subacute protocol (mean age 52.8 years with range 22-77, 119 right-handed, 63 females, 64 right hemisphere). Patients were excluded from analysis for poor quality imaging data $(n=5),<400$ frames remaining after motion scrubbing $(\mathrm{n}=8)$, or excessive hemodynamic lags (see below, $\mathrm{n}=6$ ) leaving 113 subjects in the final analysis. Demographically matched controls $(n=31)$ were recruited and underwent the same behavioral and imaging exams (mean age 55.7 years, $\mathrm{SD}=11.5$, range $21-83$ ) in two separate scanning sessions (time point 1 and time point 2 ). Controls were matched to the study population in age, gender, handedness, and level of education. Controls were excluded based on a low number of frames after motion scrubbing $(n=4$ at time point $1, \mathrm{n}=6$ at time point 2 ), leaving 26 controls at time point 1 and 25 controls at time point 2 .

Neuropsychological evaluation. Participants underwent a behavioral battery devised to assess motor, language, attention, memory, and visual function following each scanning session (details can be found in Siegel et al., 2016b). As described in Corbetta et al. 2015, principal components analysis was performed on all tests within a behavioral domain to produce a single score that predicted the highest percentage of variance across tasks. The left/right 'Motor' scores described left/right body motor performance that correlated across shoulder flexion, wrist extension/flexion, ankle flexion, hand dynamometer, nine-hole peg, action research arm test, timed walk, functional independence measure, and the lower extremity motricity index. The 'Visual Field Attention' score described contra-lesional attention biases in Posner, Mesulam, and behavioral inattention center-of-cancellation tasks. The 'Sustained Attention' score loaded on non-spatial measures of overall performance, reaction time, and accuracy on the same tests. The 'Shifting Attention' score loaded on tests indexing attention shifts, e.g. the difference in response times for attended versus unattended targets. The 'Spatial Memory' score loaded on the Brief Visuospatial Memory Test and spatial span. The 'Verbal Memory' score loaded on the Hopkins Verbal Learning Test. The 'Language' score loaded on tests devised to assess language comprehension (complex ideational material, commands, reading comprehension) and production (Boston naming, oral reading). The score of each of the seven factors for each patient was normalized using the mean and standard deviation of the corresponding factor scores in age-matched controls.

Brain imaging acquisition. Patients were scanned two weeks (mean = 13.4 days, $\mathrm{SD}=4.8$ days) after stroke onset. Controls were scanned twice at an interval of 3-months. All imaging was performed using a Siemens $3 \mathrm{~T}$ Tim-Trio scanner at the Washington University School of Medicine (WUSM) and a standard 12-channel head coil. The MRI protocol included structural, functional, pulsed arterial spin labeling (PASL), and diffusion tensor scans. Structural scans included: i) a sagitta $1 \mathrm{~T} 1$-weighted MP-RAGE $(\mathrm{TR}=1950 \mathrm{msec}, \mathrm{TE}=2.26 \mathrm{msec}$, flip angle $=$ $90^{\circ}$, voxel size $=1.0 \times 1.0 \times 1.0 \mathrm{~mm}$ ); ii) a transverse T2-weighted turbo spin-echo $(\mathrm{TR}=2500 \mathrm{msec}, \mathrm{TE}=435 \mathrm{msec}$, voxel- size $=1.0 \times 1.0 \times 1.0$ $\mathrm{mm}$ ); and iii) sagittal FLAIR (fluid attenuated inversion recovery) with $\mathrm{TR}=7500 \mathrm{msec}, \mathrm{TE}=326 \mathrm{msec}$ and voxel-size $=1.5 \times 1.5 \times 1.5 \mathrm{~mm}$. Resting-state functional scans were acquired with a gradient echo EPI sequence $(\mathrm{TR}=2000 \mathrm{msec}$, $\mathrm{TE}=27 \mathrm{msec}, 32$ contiguous $4 \mathrm{~mm}$ slices, $4 \times 4 \mathrm{~mm}$ in-plane resolution) during which participants were instructed to fixate a small white cross centered on a screen with a black background in a low luminance environment. Six to eight resting state fMRI runs, each including 128 volumes (for a total of $30 \mathrm{~min}$ ) were acquired. A camera fixated on the eyes was used to determine when a subject's eyes were open or closed during scans. Patients had eyes open on $65.6 \pm$ $31.9 \%$ of frames and controls had eyes open on $76.8 \pm 30.2 \%$ of frames (t $(114)=-1.7, \mathrm{p}=0.091)$.

Brain lesion masking. Lesions were manually segmented on individual structural MRI images (T1-weighted MP-RAGE, T2-weighted spin echo images, and FLAIR images obtained from 1 to 3 weeks post-stroke) using the Analyze biomedical imaging software system (www.mayo.edu; Robb and Hanson, 1991). Two board-certified neurologists (Dr. Maurizio Corbetta and Dr. Alexandre Carter) reviewed all segmentations. In hemorrhagic strokes, edema was included in the lesion. A neurologist (MC) reviewed all segmentations a second time paying special attention to the borders of the lesions and degree of white matter disease. Atlasregistered segmented lesions ranged from $0.02 \mathrm{~cm}^{3}$ to $82.97 \mathrm{~cm}^{3}$ with a mean of $10.15 \mathrm{~cm}^{3}\left(\mathrm{SD}=13.94 \mathrm{~cm}^{3}\right)$. Lesions were summed to display the number of patients with structural damage for each voxel.

fMRI data preprocessing. Preprocessing of fMRI data included: i) compensation for asynchronous slice acquisition using sinc interpolation; ii) elimination of odd/even slice intensity differences resulting from interleaved acquisition; iii) whole brain intensity normalization to achieve a mode value of 1000; iv) removal of distortion using synthetic field map estimation and spatial realignment within and across fMRI runs; v) resampling to $3 \mathrm{~mm}$ cubic voxels in atlas space including realignment and atlas transformation in one resampling step. Crossmodal (e.g., T2-weighted to T1-weighted) image registration was accomplished by aligning image gradients. Cross-modal image registration in patients was checked by comparing the optimized voxel similarity measure to the 97.5 percentile obtained in the control group. In some cases, structural images were substituted across sessions to improve the quality of registration. Following cross-modal registration, data were passed through three additional preprocessing steps. First, tissue-based regressors were computed based on FreeSurfer segmentation (Fischl et al., 1999a). The following sources of spurious variance were removed by regression: i) six parameters obtained by rigid body correction of head motion; ii) the signal averaged over the whole brain; iii) signal from ventricles and CSF; iv) signal from white matter. For Undirected Functional Connectivity (UFC) computations, we additionally regressed v) the average signal for gray matter. This step, commonly called global signal regression (GSR) was not applied for Granger causality (GC) computations. The rationale for this choice was to avoid any potential suppression of highly variable signals (Nalci et al., 2019) and distortion of information flow estimates using GC. Second, we performed temporal filtering retaining frequencies in the $0.009-0.08 \mathrm{~Hz}$ band. Third, we applied frame censoring meaning that the first four frames of each BOLD run were excluded. Frame censoring was implemented using frame wise displacement (Power et al., 2014) with a threshold of $1 \mathrm{~mm}$. This frame-censoring criterion was uniformly 
applied to all R-fMRI data (patients and controls).

Cortical surface processing. Surface generation and processing of functional data followed procedures similar to Glasser et al. (Glasser et al., 2013), with additional consideration for cortical segmentation in stroke patients. First, anatomical surfaces were generated for each subject's T1MRI using FreeSurfer automated segmentation (Fischl et al., 1999b). This included brain extraction, segmentation, generation of white matter and pial surface, inflation of the surfaces to a sphere, and surface shape-based spherical registration to the subject's "native" surface to the fs average surface. Segmentations were manually checked for accuracy. For patients in whom the stroke disrupted automated segmentation, or registration, values within lesioned voxels were filled with normal atlas values prior to segmentation, and then masked immediately after ( 7 patients). The left and right hemispheres were then resampled to 164,000 vertices and registered to each other (Van Essen et al., 2001), and finally down-sampled to 10,242 vertices each (a combined total of 18,722 vertices after exclusion of the medial wall) for projection of functional data. Following preprocessing, BOLD data were sampled to each subject's individual surface (between white matter and pial surface) using a ribbon-constrained sampling available in Connectome Workbench (Marcus et al., 2013). Voxels with a high coefficient of variation ( 0.5 standard deviations above the mean coefficient of variation of all voxels in a $5 \mathrm{~mm}$ sigma Gaussian neighborhood) were excluded from volume to surface mapping (Glasser et al., 2013). Time courses were then smoothed along the 10,242 vertex surface using a 3 $\mathrm{mm}$ FWHM Gaussian kernel. All brain surface visualizations were generated using Connectome Workbench (Marcus et al., 2013).

Brain parcellation scheme. We used a cortical surface parcellation generated by Gordon \& Laumann and colleagues (Gordon et al., 2016). The parcellation is based on R-fMRI boundary mapping and achieves full cortical coverage and optimal region homogeneity. The parcellation includes 324 regions of interest (159 left hemisphere, 165 right hemisphere). Note that the original parcellation includes 333 regions, while here all regions $<20$ vertices (approximately $50 \mathrm{~mm}^{2}$ ) were excluded. This cutoff was arbitrarily chosen based on the assumption that parcels below this size would have unreliable signal given $4 \mathrm{~mm}$ sampling of our functional data. Notably, the parcellation was generated on 120 young adults aged $18-33$ and is applied here to adults aged $21-83$. To generate parcel-wise connectivity matrices, time courses of all vertices within a parcel were averaged. For each ROI, we defined its center-of-mass coordinates $(\bar{x}, \bar{y}, \bar{z})$ as the average of the $(x, y, z)$ coordinates of all vertices in the ROI. For each ROI, identified the homologous regions as the ROI in having the lowest distance from $(\bar{x}, \bar{y}, \bar{z})$ (i.e., the ROI closest to be symmetrically located in the opposite hemisphere).

In addition to the 324 cortical parcels, we also defined a set of 19 subcortical and cerebellar regions based on the FreeSurfer segmentation: for each hemisphere 9 regions consisting of cerebellum, thalamus, caudate, putamen, pallidum, hippocampus, amygdala, accumbens and ventral dorsal caudate, plus brainstem (Fischl et al., 2002).

\subsection{Granger causality analysis and inter-areal information transfer}

Granger Causality (GC) framework. One of the most successful datadriven methods to quantify the degree of communication from statistical dependencies between neural signals is based on the Wiener-Granger causality principle (Granger, 1980; Brovelli et al., 2004; Ding et al., 2006; Bressler and Seth, 2011; Seth et al., 2015). In order to describe the Granger causality framework, let us consider two (discrete) time series $X=\left\{X_{t}\right\}, Y=\left\{Y_{t}\right\}$ representing the activity of two subsystems sampled at discrete times $t=\{1,2,3, \ldots, n\}$, where we assume that times are measured in units of the sampling time TR. Standard undirected functional connectivity (UFC) is classically computed as the Pearson's correlation, defined as $R=\frac{\sigma(X, Y)}{\sigma(X) \sigma(Y)}$ where $\sigma(X)$ and $\sigma(Y)$ are respectively the standard deviations of $X_{t}$ and $Y_{t}$ and $\sigma^{2}(X, Y)$ is their covariance within the selected time window. The UFC only considers dependencies between $X_{t}$ and $Y_{t}$ for the same $t$. Information-theoretically, this type of dependency is quantified by the mutual information $I\left(X_{t} ; Y_{t}\right)$, which is a simple function of $R$ for Gaussian data, $I\left(X_{t} ; Y_{t}\right)=-1 / 2 \log \left(1-R^{2}\right)$. The UFC is insensitive to the temporal structure of correlation between $X$ and $Y$, since it is invariant under permutation of $t$. On the other hand, the framework based on Granger causality (Granger, 1969; Granger, 1980) and further developed by Geweke (Geweke, 1982) considers dependencies between two time series and their "lagged" versions with different lags $1,2, \ldots$ Let us assume that $L$ is the maximum lag at which dependence is observed: in other words, $X_{t}, Y_{t}$ are not dependent on $X_{\tau}$, $Y_{\tau} \cdots$ for $\tau<t-L$, i.e., he values of $X$ and $Y$ occurring before a time $t-L$ in the past. One can thus restrict attention to dependencies between $X_{t}, Y_{t}$ and the $L$ preceding values of the time series,

$X_{t-1}^{(L)} \equiv X_{t-1}, X_{t-2}, \cdots, X_{t-L}$ and $Y_{t-1}^{(L)} \equiv Y_{t-1}, Y_{t-2}, \cdots, Y_{t-L}$

Interdependencies between the two time series reflected into the fact that the time series of $Y$ contains information about $X_{t}$, and vice versa. The total amount of information about $X_{t}$ contained in $Y$ can be quantified by the mutual information between $X_{t}$ and $Y$ (present and past): $I\left(X_{t} ; Y_{t}, Y_{t-1}^{(L)}\right)$. By virtue of the identity $I(A ; B C)=I(A ; B)+I(A ; C \mid B)$, this quantity can be decomposed into an "instantaneous" and a "lagged" term:

$I\left(X_{t} ; Y_{t}, Y_{t-1}^{(L)}\right)=I\left(X_{t} ; Y_{t} \mid Y_{t-1}^{(L)}\right)+I\left(X_{t} ; Y_{t-1}^{(L)}\right)$

The conditioning on the instantaneous term implies that $I\left(X_{t} ; Y_{t} \mid Y_{t-1}^{(L)}\right)$ measures information about $X_{t}$ contained exclusively in $Y_{t}$ (and not already contained in $Y_{t-1}^{(L)}$ ).

The basic idea of Granger causality is that $Y$ contains exclusive information about $X_{t}$,

which is not already present in the time series of $X$, i.e., in $X_{t-1}^{(L)}$. To obtain the "exclusive" information about $X_{t}$ contained in $Y$ one should further condition over $X_{t-1}^{(L)}$ :

$I\left(X_{t} ; Y_{t}, Y_{t-1}^{(L)} \mid X_{t-1}^{(L)}\right)$

and again obtain an "instantaneous" and a "lagged" term:

$I\left(X_{t} ; Y_{t}, Y_{t-1}^{(L)} \mid X_{t-1}^{(L)}\right)=I\left(X_{t} ; Y_{t} \mid X_{t-1}^{(L)}\right)+I\left(X_{t} ; Y_{t-1}^{(L)} \mid X_{t-1}^{(L)}\right)$

The first term is called instantaneous causality (IC) and usually indicated by $F_{X \cdot Y}$

$F_{X \cdot Y} \equiv I\left(X_{t} ; Y_{t} \mid X_{t-1}^{(L)}, Y_{t-1}^{(L)}\right)$

The second term is called directed causality (DC) from $Y$ to $X$ and usually indicated by $F_{Y \rightarrow X}$

$F_{Y \rightarrow X} \equiv I\left(X_{t} ; Y_{t-1}^{(L)} \mid X_{t-1}^{(L)}\right)$

Symmetrically, the exclusive information about $Y_{t}$ contained in $X$ is measured by

$I\left(Y_{t} ; X_{t}, X_{t-1}^{(L)} \mid Y_{t-1}^{(L)}\right)=I\left(X_{t} ; Y_{t} \mid X_{t-1}^{(L)}, Y_{t-1}^{(L)}\right)+I\left(Y_{t} ; X_{t-1}^{(L)} \mid Y_{t-1}^{(L)}\right)$

The first term coincides with $F_{X \cdot Y}$ and the second one is the directed causality from $X$ to $Y$,

$F_{X \rightarrow Y} \equiv I\left(Y_{t} ; X_{t-1}^{(L)} \mid Y_{t-1}^{(L)}\right)$

The measures $F_{X \rightarrow Y}, F_{Y \rightarrow X}$ and $F_{X \cdot Y}$ were proposed by Geweke (Geweke, 1982), who also defined the total interdependence between $X$ and $Y$ as the sum of all the three terms,

$F_{X, Y}=F_{X \rightarrow Y}+F_{Y \rightarrow X}+F_{X \cdot Y}$

This is the "new" dependency between $X$ and $Y$ "created" at each timet, indeed

$F_{X, Y}=I\left(X_{t}, X_{t-1}^{(L)} ; Y_{t}, Y_{t-1}^{(L)}\right)-I\left(X_{t-1}^{(L)} ; Y_{t-1}^{(L)}\right)$ 
Thus, in the GC framework, the total interdependence between two signals can be split into three terms: two directed Granger causality (DC) terms and an instantaneous Granger causality (IC) term. The DC terms $\left(F_{X \rightarrow Y}, F_{Y \rightarrow X}\right)$ represent a directed flow of information from $X$ to $Y$ or vice versa, occurring over a timescale $>1$ TR (Fig. 1b). The IC term $\left(F_{X \cdot Y}\right)$ represents information shared between $X$ and $Y$ "instantaneously", i.e., in less than one TR, and it accounts for direct communication, or unconsidered influences that may originate from common (e.g., subcortical) sources, occurring in less then one TR (Fig. 1a).

Granger causality measures can therefore be formulated in completely information-theoretical terms (Barnett et al., 2009; Marko, 1973; Rissanen and Wax, 1987; Schreiber, 2000). Information-theoretic measures based on the Wiener-Granger principle, such as Transfer Entropy (Schreiber, 2000) and Directed Information (Massey, 1990), represent the most general measures of Wiener-Granger causality and capture any (linear and nonlinear) time-lagged conditional dependence between neural signals (Besserve et al., 2015; Vicente et al., 2011).

Covariance-based Granger Causality. The GC measures $F_{X \rightarrow Y}, F_{Y \rightarrow X}$ and $F_{X \cdot Y}$ capture statistical relations among the values of $X, Y$ in a time window of length $L+1$ including the "present" values $X_{t}, Y_{t}$, and the "past" values $X_{t-1}^{(L)}, Y_{t-1}^{(L)}$. Together, $X_{t}, Y_{t}, X_{t-1}^{(L)}, Y_{t-1}^{(L)}$ define a vector of length $2 L+2$ values. The GC measures can be ultimately expressed in terms of Shannon entropies involving the $(2 L+2$-variate) probability distribution $P\left(X_{t}, Y_{t}, X_{t-1}^{(L)}, Y_{t-1}^{(L)}\right)$ and some of its marginals:

$F_{X \rightarrow Y}=H\left(Y_{t}, Y_{t-1}^{(L)}\right)-H\left(Y_{t-1}^{(L)}\right)-H\left(Y_{t}, X_{t-1}^{(L)}, Y_{t-1}^{(L)}\right)+H\left(X_{t-1}^{(L)}, Y_{t-1}^{(L)}\right)$

$F_{X \cdot Y}=H\left(Y_{t}, X_{t-1}^{(L)}\right)+H\left(X_{t}, X_{t-1}^{(L)}, Y_{t-1}^{(L)}\right)-H\left(X_{t-1}^{(L)}, Y_{t-1}^{(L)}\right)-H\left(X_{t}, Y_{t}, X_{t-1}^{(L)}, Y_{t-1}^{(L)}\right)$

Assuming the distribution $P\left(X_{t}, Y_{t}, X_{t-1}^{(L)}, Y_{t-1}^{(L)}\right)$ to be stationary, the classical method to compute entropies is the "binning method". One considers $T$ running windows of length $L+1$, and for each window extracts the vector $X_{t}, Y_{t}, X_{t-1}^{(L)}, Y_{t-1}^{(L)}$, thus obtaining $T$ samples of $2 L+2$. Binning each univariate variable and collecting the bin counts, the joint probability distribution $P\left(X_{t}, Y_{t}, X_{t-1}^{(L)}, Y_{t-1}^{(L)}\right)$ is approximated by (multidimensional) histogram (Beirlant et al., 1997; Treves and Panzeri, 1995). If $n$ bins are used for each univariate variable, the total number of multidimensional bins is $n^{2(L+1)}$. As a rule of thumb, to get at least a rough estimate of the bin counts one needs at least as many samples as bins, so $T \geq n^{2(L+1)}$ points. Since $L \geq 1$, this requires a large sample $T \geq$ $n^{4}$ for estimation. In order to make the estimation feasible on short time windows, a common solution is to approximate the distribution with the

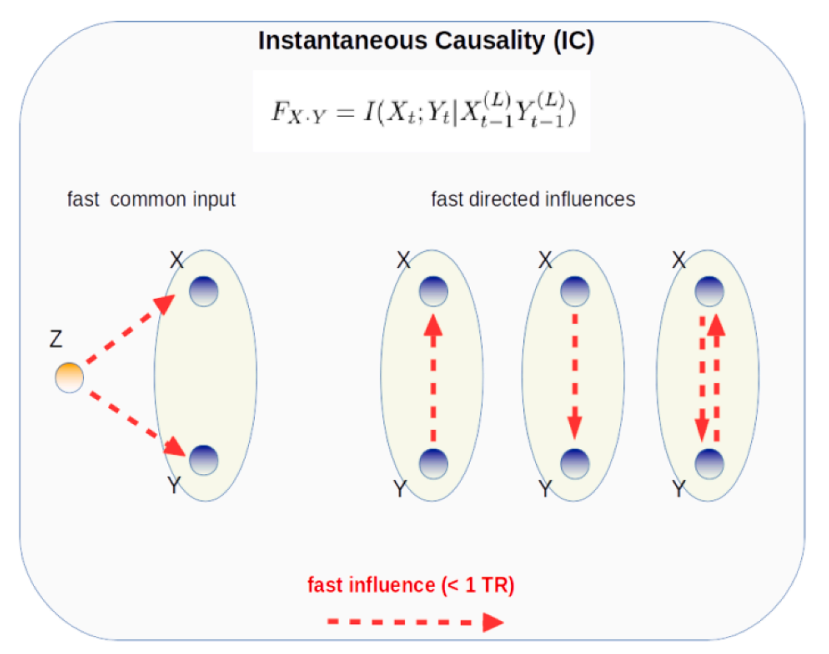

(a) first term of the Gram-Charlier expansion, i.e., by a Gaussian distribution with the same second order moments (covariance matrix) as the given distribution. This approximation amounts to keeping only second order statistics, and neglecting higher-order terms, and is relatively accurate for fMRI data (Hlinka et al., 2011). In this approximation, the distribution $P\left(X_{t}, Y_{t}, X_{t-1}^{(L)}, Y_{t-1}^{(L)}\right)$ and its marginals are effectively replaced by the covariance matrix $\Sigma\left(X_{t}, Y_{t}, X_{t-1}^{(L)}, Y_{t-1}^{(L)}\right)$ and its submatrices. Estimating $\Sigma$ requires only to estimate $(2 L+2)^{2}$ parameters corresponding to the second moments of the distribution. Furthemore, entropies can be simply computed with the formula

$H(A)=\frac{n_{A}}{2} \log 2 \pi e+\frac{1}{2} \log |\Sigma(A)|$

where $\Sigma(A)$ is the covariance matrix of $A, n_{A}$ the dimension of $A$, and $|\cdot|$ is the determinant. In this covariance-based approximation, GC measures are expressed in terms of determinants of submatrices of the covariance matrix of the data (Brovelli et al., 2015). For instance,

$F_{X \rightarrow Y}=\frac{1}{2} \log \left|\Sigma\left(Y_{t}, Y_{t-1}^{(L)}\right)\right|-\frac{1}{2} \log \left|\Sigma\left(Y_{t}\right)+\right|$

$\left.-\frac{1}{2} \log \left|\Sigma\left(Y_{t}, X_{t-1}^{(L)}, Y_{t-1}^{(L)}\right)\right|+\frac{1}{2} \log \mid \Sigma\left(X_{t-1}^{(L)}, Y_{t-1}^{(L)}\right)\right)$

The covariance-based GC estimation is equivalent to the parametric estimation of GC from an autoregressive model with Gaussian innovations, i.e., the traditional way to estimate Granger causality.

Gaussian-copula-based estimation of GC. The GC measures $F_{X \rightarrow Y}$, $F_{Y \rightarrow X}$ and $F_{X \cdot Y}$ can all be written as appropriate sums of mutual information (MI) terms. For instance,

$F_{X \rightarrow Y}=I\left(Y_{t} ; X_{t-1}^{(L)} \mid Y_{t-1}^{(L)}\right)=I\left(Y_{t} ; X_{t-1}^{(L)}, Y_{t-1}^{(L)}\right)-I\left(Y_{t} ; Y_{t-1}^{(L)}\right)$

The MI is invariant under monotonic transformations of the marginals, and this fact can be exploited to relax the assumption of Gaussianity. In particular, one can replace the assumption of Gaussianity with the weaker assumption of a Gaussian copula (Ince et al., 2017): that the joint distribution of the variables can be rendered Gaussian by means of local transformations on the marginals. Formally, consider the transformations

$X=t(X), Y=u(Y)$

where $t, u$ are monotonic functions. The Gaussian copula assumption is equivalent to the existence of $t, u$ such that $P(X, Y)$ is Gaussian. While Gaussianity imposes a linear dependence between variables, the

$$
F_{Y \rightarrow X}=I\left(X_{t} ; Y_{t-1}^{(L)} \mid X_{t-1}^{(L)}\right) \quad F_{X \rightarrow Y}=I\left(Y_{t} ; X_{t-1}^{(L)} \mid Y_{t-1}^{(L)}\right)
$$

slow directed influence slow directed influence
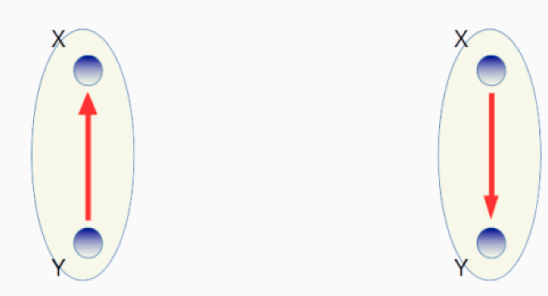

slow influence $(>1 \mathrm{TR})$

Fig. 1. Interpretation of instantaneous and directed Granger causality in terms of information flows between two areas. 
Gaussian copula assumption allows for more general monotonic dependence (Ince et al., 2017). Under the Gaussian copula assumption, there is a simple way to compute the MI. Since the MI is invariant under monotonic transformations of the marginals, $I(X ; Y)=I(X:)$, and since there exist $X, Y$ such that $P(X, Y)$ is Gaussian, it is sufficient to find $X$, $Y$ and compute $I(X ; Y)$ with the covariance-based formula (12), which is exact for $X, Y$.

Finding $X, Y$ is easy. Consider two random variables $X, Y$ with joint cumulative distribution function (CDF) $H(X, Y)$ and marginal CDFs $F(X)$, $G(X)$. Consider

$t(X)=\Phi^{-1}(F(X)), u(Y)=\Phi^{-1}(G(Y))$

where $\Phi$ is the CDF of a standard normal variable. One can immediately show that $X=t(X), Y=u(Y)$ are standard normal variables, i.e., $F(X)=$ $\Phi(X), G(Y)=\Phi(Y)$. Applying the covariance-based approach to the transformed variables, one has $I(X ; Y)=\frac{1}{2} \log \frac{\left|\Sigma_{X}\right|\left|\Sigma_{Y}\right|}{\left|\Sigma_{X Y}\right|}$, and by virtue of the invariance $I(X ; Y)=I(X ; Y)$.

The Gaussian copula evaluation of the MI is not exact. In general, even though the marginal distribution of $X, Y$ are Gaussian, the joint distribution is not perfectly a bivariate Gaussian. This is true only if the copula, i.e., the part of the distribution specifying the dependence between the two variables, is Gaussian (see Ince et al. 2017 for a definition of copula and discussion of this point). However, for many distributions the Gaussian copula assumption is approximately met. In summary, one obtains the following algorithm to compute the MI: i) given samples $\left\{X_{i}\right.$, $\left.Y_{i}\right\}$, approximate $F(X), G(Y)$ with the empirical CDFs $F\left(X_{i}\right)=$ $\operatorname{rank}\left(X_{i}\right) / N, G\left(Y_{i}\right)=\operatorname{rank}\left(Y_{i}\right) / N$ and compute $t\left(X_{i}\right)=\Phi^{-1}\left(\operatorname{rank}\left(X_{i}\right) / N\right)$, $u\left(Y_{i}\right)=\Phi^{-1}\left(\operatorname{rank}\left(Y_{i}\right) / N\right) \cdot t\left(X_{i}\right)$ and $u\left(Y_{i}\right)$ are normally distributed and have the same MI as $X_{i}, Y_{i}$ ii) compute the MI from the samples $\left\{t\left(X_{i}\right)\right.$, $\left.u\left(Y_{i}\right)\right\}$ with the covariance-based method. In our work, we have computed all GC measures by expressing them in terms of sums of MIs and then applying the Gaussian-copula-based estimation to each term in the sum.

Relation between linear correlation and covariance-based Granger causality measures. Standard undirected functional connectivity (UFC) is classically computed as the Pearson's correlation, defined as $R(X, Y)=$ $\frac{\sigma(X, Y)}{\sigma(X) \sigma(Y)}$ where $\sigma(X), \sigma(Y)$ are the standard deviations of $X_{t}$ and $Y_{t}$ and $\sigma^{2}(X, Y)$ is their covariance within the selected time window. The UFC only considers dependencies between $X_{t}$ and $Y_{t}$ for the same $t$. UFC and covariance-based Granger causality measures share common properties. Linear correlation and total interdependence are undirected measures quantifying static and dynamic dependencies, respectively. Although these measures are not related by a mathematical decomposition, there is a strong relationship between the existence of both types of dependencies. A lack of total interdependence implies a lack of linear correlation; and, if we assume that the future of $X$ and $Y$ causally depends on their own past, respectively, the opposite relation is also true. This occurs because linear correlation is related to the covariance-based approximation of the mutual information, $I\left(X_{t} ; Y_{t}\right)=-1 / 2 \log \left(1-R^{2}\right)$, and because conditioning on the past cannot create new dependencies (Chicharro and Ledberg, 2012). It is also clear the directed and instantaneous Granger measures are smaller than the total interdependence. Thus, null total interdependence implies the absence of Granger causality measures because they constitute non-negative contributions to the total interdependence. In other words, Granger causality is present if, and only if, both linear correlation and total Granger interdependencies are not zero.

The FC and GC quality-based exclusion criteria. In order to ensure good-quality FC and GC estimates, we excluded from analysis all subjects with $<400$ usable frames after motion scrubbing. Furthermore, for each subject, we computed a lag between homologous ROIs as in (Siegel et al., 2016b). In brief, for any integer lag $l=-4,-3, \cdots, 3,4$ we computed the lagged cross-correlation $C_{l}=\left\langle X_{t} Y_{t+l}\right\rangle$ between the
BOLD signals $X, Y$ of the homologous ROIs; the homotopic lag between the ROIs was identified by finding $l_{0}=\operatorname{argmin}\left(C_{l}\right)$, performing a parabolic interpolation on $C_{l_{0}-1}, C_{l_{0}}, C_{l_{0}+1}$, and computing the minimum of the parabola. An average homotopic lag between the left and right hemisphere was computed by averaging over all homptipic lags between left ROIs and the homologous right ROIs. Anomalously large homotopic lags are a likely indication of the presence of lags of hemodynamic origin, due to disruption of the standard hemodynamic response in the vicinity of the lesion. Therefore, we excluded from analysis all subjects with severe homotopic lags ( $>1 \mathrm{~s}$ inter-hemispheric difference). After motion and lag exclusion, 113 patients were included at two weeks, 27 controls at time point one, and 25 at time point two.

\subsection{Outline of the analyses}

Our main goal is to characterize the main changes in inter- and intrahemispheric communication in stroke. The analyses are designed to optimally highlight such changes at the group level, and at the level of resting-state networks (i.e., not at the single-subject, single-region level).

Resting state networks. As in Gordon et al., 2016, each of the 343 regions considered can be labeled as belonging to one of twelve cortical resting state networks (RSNs), or to the set of subcortical regions, or to the set of cortical regions with no network affiliation ("None" affiliation in Gordon et al., 2016). To simplify the analysis, we excluded from analysis those cortical regions belonging to either small networks (with $<5$ nodes) or to no network (the ("None" affiliation in Gordon et al., 2016, which mostly comprises ventral regions belonging to the limbic system). We thus considered the following nine RSNs: the visual network (VIS), sensorimotor dorsal network (SMD), sensorimotor ventral network (SMV), auditory network (AUD), cingulo-opercular network (CON), ventral attention network (VAN), dorsal attention network (DAN), default mode network (DMN), fronto-parietal network (FPN). In addition, we considered 19 subcortical and cerebellar regions (SUB). The following cortical networks were left out of analysis: retrosplenial network (5 nodes), cingulo-parietal network (4 nodes), salience network (5 nodes).

Network-wise measures of inter-and intra-hemispheric connectivity. We will first evaluate undirected functional connectivity (UFC), instantaneous Granger Causality (IC) and directed Granger Causality (DC) at the level of single subjects, single regions, obtaining individual $343 \times 343$ connectivity matrices. For analysis, however, we will focus on summary measures quantifying inter- and intra-hemispheric connectivity at the network level:

To assess inter-hemispheric connectivity, we will focus on homotopic connections linking homologous ROIs located symmetrically in opposite hemispheres. We will thus compute the UFC $R(X, Y)$, the IC $F_{X \cdot Y}$ and the DC $F_{X \rightarrow Y}, F_{Y \rightarrow X}$ where $X$ and $Y$ are homologous ROIs. For convenience, we will choose $X$ in the healthy hemisphere and $Y$ the homologous ROI in the lesioned hemisphere, so that $F_{X \rightarrow Y}, F_{Y \rightarrow X}$ will represent connectivity from the healthy to the lesioned hemisphere and from the lesioned to the healthy hemisphere, respectively. For healthy subjects, the healthy and lesioned hemisphere will be replaced by the left (dominant) and right (non-dominant) hemisphere. Once UFC, IC and DC have been computed for all homotopic pairs, we will average over all pairs belonging to each of the 10 considered networks. Thus, for each subject, we will have $10 \times 1$ arrays of network-wise homotopic UFC, IC and DC. As for DC we will have two arrays, one from the healthy to the lesioned hemisphere, and one from the lesioned to the healthy hemisphere.

To assess intra-hemispheric connectivity, we will compute the $\operatorname{UFCR}(X, Y)$, the IC $F_{X \cdot Y}$ and the DC $F_{X \rightarrow Y}, F_{Y \rightarrow X}$ where $X$ and $Y$ are regions located in the same hemisphere (healthy/lesioned for patients, left/right for controls). Once UFC, IC and DC have been computed for all intrahemispheric pairs, we compute network-wise measures by considering all pairs of nodes where either $X$ or $Y$ belongs to the given network and averaging. Thus, for each network we considered the sum of its con- 
nections with all networks. For each subject, we will have $10 \times 1$ arrays of network-wise intrahemispheric UFC, IC and DC, one for the lesioned hemisphere and one for the healthy hemisphere.

Statistical analyses. We will compare results at the group level. Mainly, we will compare healthy participants $(n=26)$ and stroke patients $(\mathrm{n}=113)$. We will also compare patients with left hemisphere lesions ( $\mathrm{LH}, \mathrm{n}=60)$ and right hemisphere lesions $(\mathrm{RH}, \mathrm{n}=53$ ). Statistical comparisons will be systematically performed with ANOVAs with group and RSN as factors, and hemisphere (lesioned/healthy) as a third factor when appropriate. When network is a significant factor (or yields significant network $\times$ group, network $\times$ hemisphere interactions) we will additionally perform post-hoc T-tests to highlight the effect of specific RSNs. In particular, we will perform the following analyses:

Two-way ANOVA on homotopic UFC with RSN and group (patients/ controls) as factors. Same for homotopic IC.

Three-way ANOVA on homotopic DC with RSN, group (patients $v s$. controls) and hemisphere (lesioned to healthy $v s$. healthy to lesiones) as factors.

Three-way ANOVA on intrahemispheric UFC with RSN, group (patients $v s$. controls) and hemisphere (lesioned $v s$. healthy) as factors. Same for intrahemispheric IC, DC.

Once significant effects distinguishing patients from controls are found, we will focus on the group of patients, investigating whether $\mathrm{LH}$ and $\mathrm{RH}$ patients present differences in the magnitude of the observed effects.

Finally, we will analyze the relation between connectivity measures and behavioral impairment. Once effects distinguishing patients and controls at a group level are identified, we will consider the corresponding measures at an individual level and correlate them with behavioral scores. We will use eight behavioral scores identified in a previous work (Corbetta et al., 2015), corresponding to the eight strongest principal components explaining a large fraction of variance in behavioral tests covering language, memory, motion and attention function. For example, if we find that homotopic IC is reduced in the group of patients compared to controls, we will correlate the individual homotopic UFC with individual behavioral scores, to investigate whether the functional anomaly is related to behavioral impairment.

\section{Results}

We analyzed resting-state fMRI data recorded from acute stroke patients $(n=113)$ and healthy participants $(n=26)$. Our analysis tested the hypothesis that post-stroke FC alterations are tightly intertwined with information flow deficits occurring both inter-hemispherically and intra-hemispherically. To address this issue, we performed covariancebased Granger causality (GC) analyses (Brovelli et al., 2015) of resting-state fMRI data and compared inter-areal information flow analyses with standard FC approaches. The comparison was performed by means of the notion of total interdependence between signals (Geweke, 1982). In the GC framework, the total interdependence (TI) between two signals can be split into three terms: two directed Granger causality (DC) terms and an instantaneous Granger causality (IC) term. The DC terms $\left(F_{X \rightarrow Y}, F_{Y \rightarrow X}\right)$ represent a directed flow of information from $X$ to $Y$ or vice versa, occurring over a timescale $>1$ TR (Fig. 1b). The IC term $\left(F_{X \cdot Y}\right)$ represents information shared between $X$ and $Y$ "instantaneously", i.e., in less than one TR, and it accounts for rapid direct communication, or unconsidered influences that may originate from common (e.g., subcortical) sources (Fig. 1a). Functional MRI data were computed for 324 parcels of the Gordon-Laumann cortical parcellation (Gordon et al., 2016) and 19 sub-cortical and cerebellar parcels from the FreeSurfer atlas (Fischl, 2012). For each subject and for each pair of parcels, or regions-of-interest (ROIs), we evaluated the undirected functional connectivity (UFC, z-transformed Pearson correlation), the instantaneous Granger causality (IC) and the directed Granger causality in both directions (DC). For healthy controls, the DC, IC and UFC matrices obtained in two independent sessions were averaged for subjects $(n=24)$ for which they were both available.

\subsection{Consistency of UFC and GC measures}

We first tested the reliability of our results by verifying the consistency of the UFC and GC measures obtained for control participants in two separate sessions (Fig. 2a). Consistency was defined as the Pearson correlation between the (upper-triangular parts of the) corresponding matrices in the two sessions. The UFC matrices were moderately consistent ( $r=0.65 \pm 0.03$, average and standard error over subjects). The same result was obtained for IC matrices $(r=0.73 \pm 0.02)$. The DC matrices, instead, were poorly consistent $(r=0.22 \pm 0.02)$. We obtained reduced network-wise $(28 \times 28)$ matrices by averaging over ROIs in the same network and hemisphere. We considered thirteen cortical restingstate networks as in (Gordon et al., 2016), plus subcortical ROIs. Consistency improved for UFC ( $r=0.80 \pm 0.03)$, IC $(r=0.87 \pm 0.03)$, and DC $(r=0.41 \pm 0.03)$. The UFC and IC results are thus reliable at the single-subject level, especially if network-averaged results are considered. As for the DC, due to the poor level of consistency obtained in the full $(343 \times 343)$ DC matrix, we cannot expect reliable results at the level of single subject, single ROI. Also at the network level individual results are not completely reliable. To assess the reliability of group results, we computed the consistency of group-averaged FC matrices for random groups of $n$ participants (Fig. 2b). The group consistency is significantly stronger than the individual consistency. When considering groups of 5 subjects, the DC consistency rises to 0.4 ( 0.7 for network-wise matrices), and for 10 subjects it rises to 0.5 ( 0.8 for network-wise matrices). This result implies that while individual DC results are affected by a very large noise, DC results at the group-level are reliable. In summary, UFC and IC matrices were highly consistent both at the individual and group level, while DC matrices were consistent only at the group level.

To better understand the relation between the degree of consistency across sessions and the variance explained by each metric, we computed the fraction of TI due to the IC and DC. In Fig. 2c, we show the proportion over the TI averaged across links for each individual subject. Overall, the IC accounts for a large fraction (mean: 70\%, s.d.: $10 \%$ ) of the TI. This fraction is even higher if we compute the mean over homotopic links, which are the strongest functional links, connecting homologous ROIs located symmetrically in opposite hemispheres (mean: 80\%, s.d.: 4\%). Another class of strong functional links is given by intra-hemispheric links within the same resting state network: also in this case, the largest fraction of the TI is due to the IC (mean: 71\%, s.d.: $6 \%$ ). If we consider weak functional links connecting different resting state networks, the fraction of TI due to the IC decreases, but remains well above $50 \%$ (mean: $61 \%$, s.d. $8 \%$ ). These results imply that most of the correlation between the time series of different ROIs is due to interactions occurring within the time resolution of fMIR $(1 \mathrm{TR}=2 \mathrm{~s})$. This limits the detectability of directed interactions (DC), and consequently also the resolvability of directionality.

Finally, we note that in our data set we do not have multiple sessions for each patient at a single clinical phase, so we cannot repeat the replicability analysis done on controls. In principle, however, the replicability of results across sessions recorded in identical conditions (such as the sessions recorded at TP1 and TP2 for controls) should depend only on the details of the recording (e.g., machine and TR) and the specific measures computed, so we would expect a similar degree of individual between-session consistency also for patients. Of course, the group consistency of patients could be significantly lower, as patients constitute a more heterogeneous sample than healthy controls. While we cannot estimate the group consistency across sessions for patients, we can assess the group consistency of results as a function of group size by comparing group results obtained with random samples of equal size (Fig. 2d). The across-group consistency of UFC, IC and DC for patients is generally comparable to the across-session group consistency obtained for healthy controls, implying that the group results for patients enjoy the same degree of reliability. 


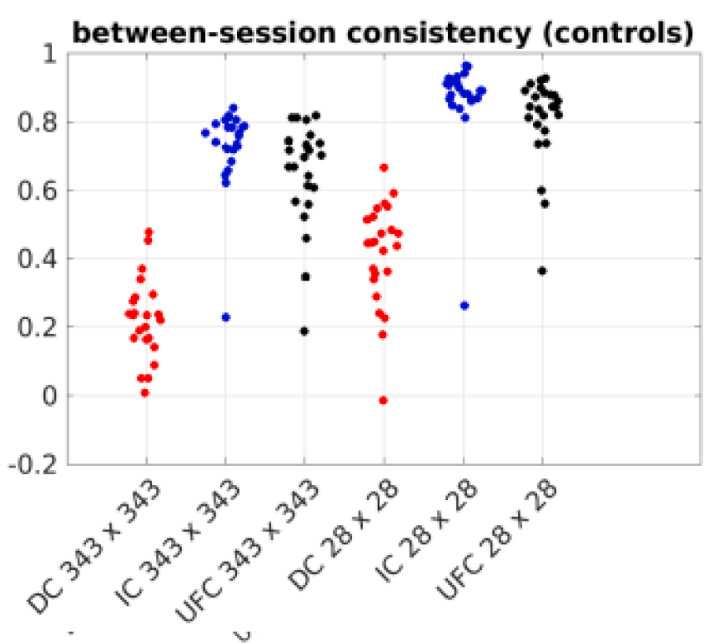

(a)

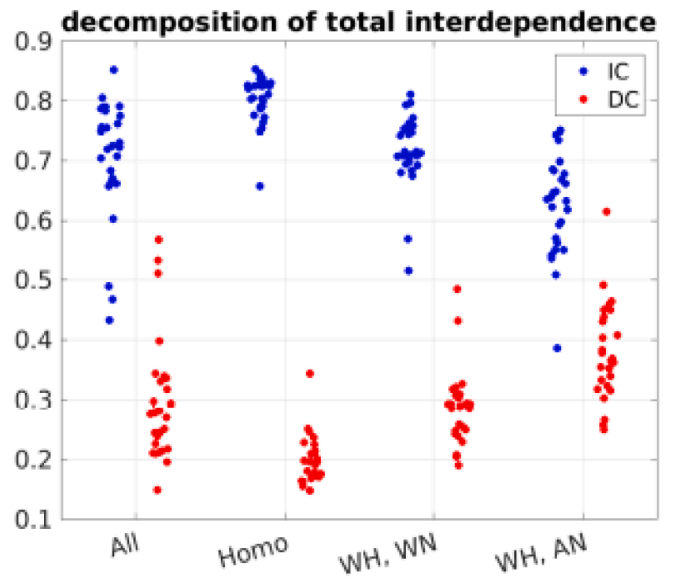

(c)

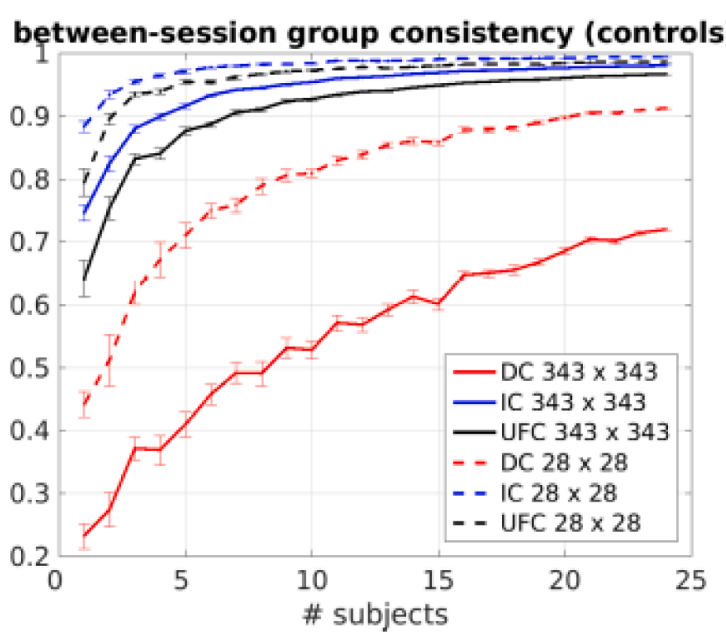

(b)

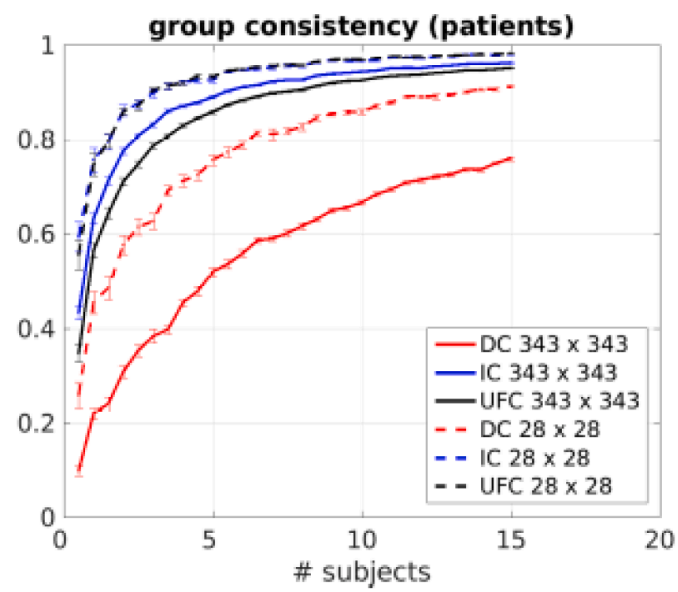

(d)

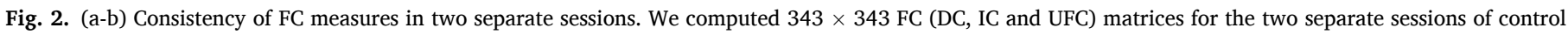

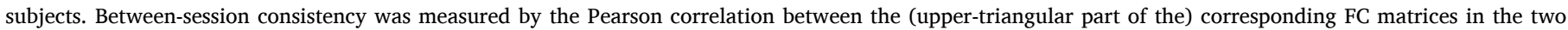

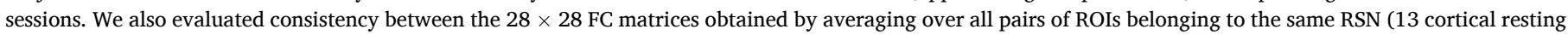

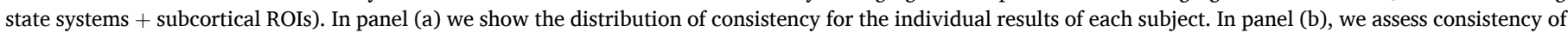

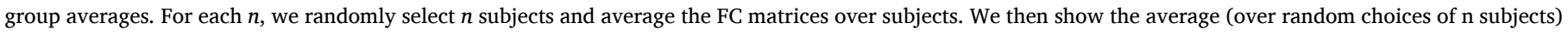

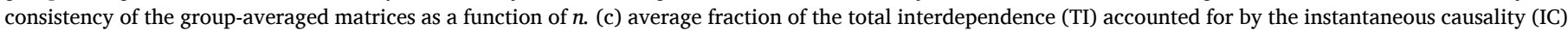

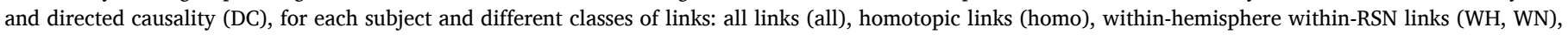

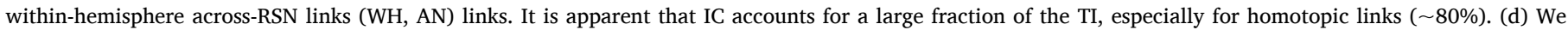

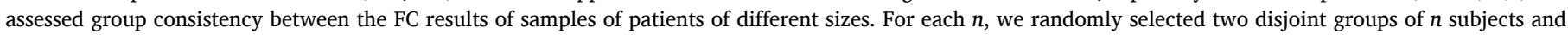

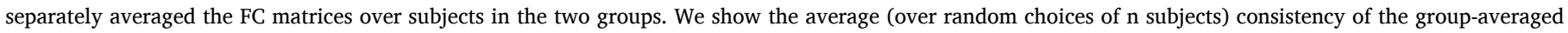
matrices as a function of $n$.

\subsection{Interhemispheric homotopic undirected functional connectivity and Granger causality analyses}

Previous studies have shown that stroke patients present a reduced interhemispheric UFC with respect to healthy controls (Carter et al., 2010; Golestani et al., 2013; He et al., 2007; New et al., 2015; Park et al., 2011; Ramsey et al., 2016; Siegel et al., 2016b; Tang et al., 2016). This effect is strongest for interhemispheric homotopic connections, which link homologous ROIs located symmetrically in opposite hemispheres. We computed UFC, IC, and DC between pairs of homologous ROIs and compared healthy controls $(C, n=26)$ with stroke patients $(P, n=113)$ in the subacute phase.

Network-wise measures of homotopic connectivity were obtained by averaging over pairs of homotopic ROIs belonging to the same resting state network (RSNs, see Methods for details). Fig. 3a shows the distribution of the homotopic UFC, averaged over all homotopic pairs belonging to each RSN. Controls had significantly higher UFC than patients in most networks, but the magnitude of the gap differed, with strongest effects in VIS, SMD, and subcortical regions (two-way repeatedmeasures ANOVA with group and network as factors; effect of group $F$ $(1,1233)=11.9, p=0.0007$; group $\times$ network interaction: $F(9,1233)=$ 2.8, $p=0.003$; post-hoc T-tests controls vs patients for each network: $p<$ 0.05 in all networks except VAN, $p<0.005$ for VIS, SMD, SUB, FDRcorrected for 10 comparisons). Analogous results were already obtained in previous analyses (Siegel et al., 2016a).

The analysis of homotopic UFC in patients showed that the activity of homologous regions is less synchronized than in healthy controls, suggesting a reduced interaction between the hemispheres. Granger causality (GC) analyses were performed to characterize instantaneous (IC) and directional (DC) interactions between homologous regions. Fig. 3b 


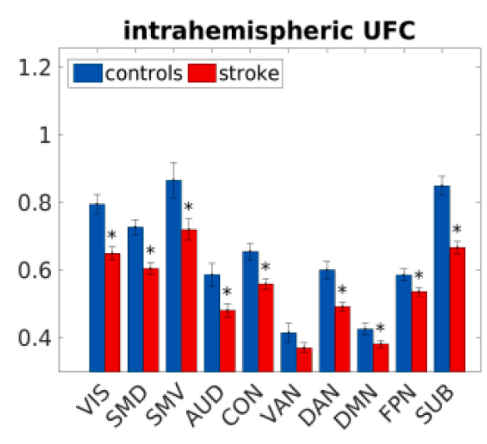

(a)

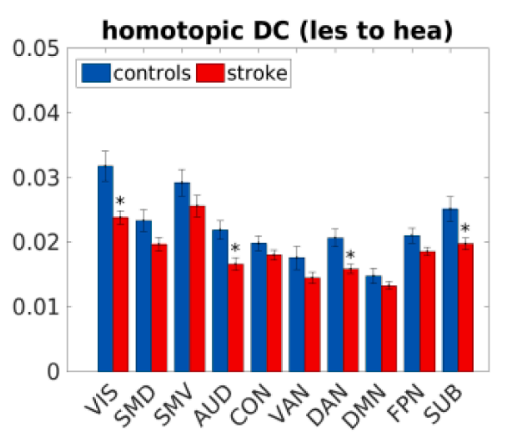

(d)

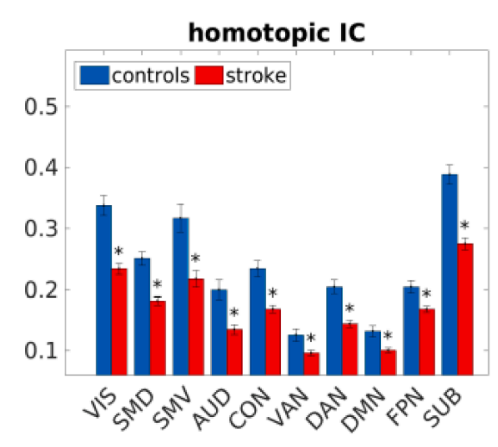

(b)

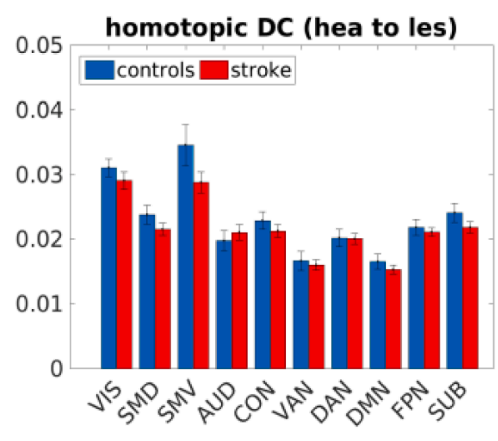

(e)

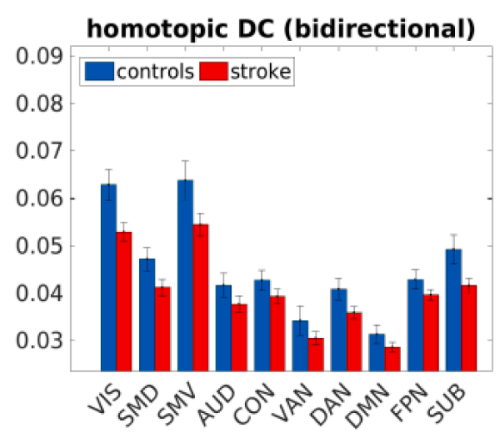

(c)

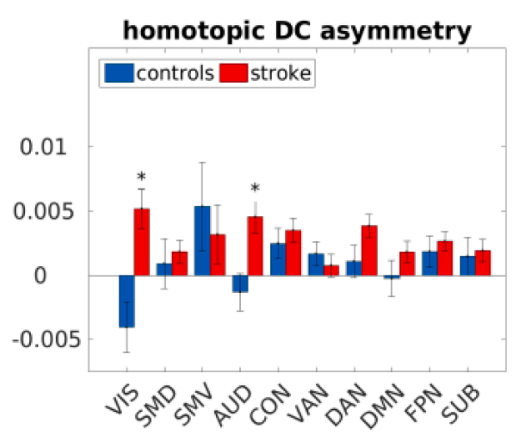

(f)

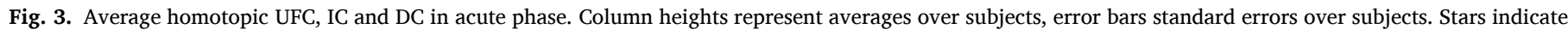

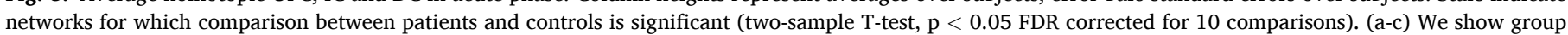

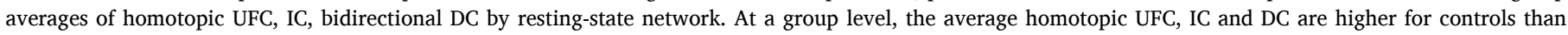

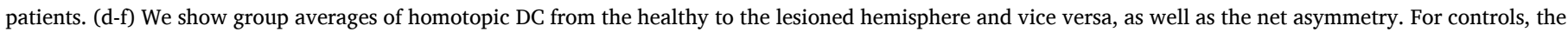

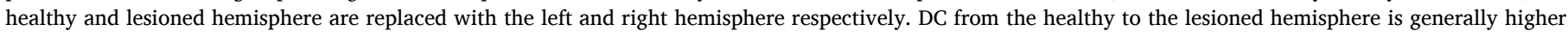
than the opposite way around, leading to a positive asymmetry.

depicts the distribution of the instantaneous causality (IC), averaged over all homotopic pairs of ROIs belonging to each RSN. Controls had significantly higher IC than both patients in all networks, but the magnitude of the gap differed, with the strongest differences in the VIS, SMD/SMV, and subcortical regions (like for UFC) but also in CON, DAN (two-way repeated-measures ANOVA with groups ad network as factors, group: $F(1,1233)=25.7, p=1 \cdot 10^{-6}$; group $\times$ network interaction: $F$ $(9,1360)=6.3, p=1.0 \cdot 10^{-8}$; post-hoc T tests controls $v$ s patients for each network, $p<0.05$ in all networks, $p<0.001$ for VIS, SMD, SMV, CON, $D A N$, SUB, FDR-corrected for 10 comparisons). In summary, the IC results were in qualitative agreement with those of UFC, but the discrepancy between patients and controls was more pronounced (as mirrored in a larger group effect and more pronounced network-wise differences).

We then analyzed directional Granger causality measures between homologous regions. The DC potentially allows revealing asymmetries between the hemispheres: we conjectured that, due to lesions in patients, homotopic information flow from the healthy to the lesioned hemisphere could be different than in the opposite direction. We analysed separately homotopic DC terms $F_{X \rightarrow Y}, F_{Y \rightarrow X}$ where $X$ is a ROI in the healthy hemisphere and $Y$ the homologous ROI in the lesioned hemisphere. For controls, we replaced the healthy hemisphere with the left (dominant) hemisphere. Fig. 3d shows the distribution of the homotopic DC from the lesioned to the healthy hemisphere, averaged over homotopic pairs of ROIs belonging to each RSN. Fig. 3e shows the distribution of homotopic DC from the healthy to the lesioned hemisphere. Overall, the homotopic information flow was reduced in patients compared to controls (three-way ANOVA with group, network and directionality as factors on homotopic DC: effect of group $F(1,137)=4.0, p=0.04)$. Moreover, the homotopic flow from the healthy to the lesioned hemisphere was higher than the reverse (effect of directionality $F(1,2603)=64.4, p<$ $\left.10^{-10}\right)$, with a stronger asymmetry in patients compared to controls (group $\times$ directionality interaction $F(9,2584)=8.7, p=0.003)$. These effects can be more easily visualized by computing the bidirectional homotopic DC, defined as the sum of DC estimates in both directions, $S_{X \leftrightarrow Y}=F_{X \rightarrow Y}+F_{Y \rightarrow X}$, as well as the total DC asymmetry $G_{X \rightarrow Y}=F_{X \rightarrow Y}-F_{Y \rightarrow X}$ where $X$ is a ROI in the healthy hemisphere and $Y$ the homologous ROI in the lesioned hemisphere. $G_{X \rightarrow Y}$ larger than zero implies a net information flow from the healthy to the lesioned hemisphere, and vice versa for $G_{X \rightarrow Y}$ smaller than zero. For controls, we computed the asymmetry $G_{X \rightarrow Y}=F_{X \rightarrow Y}-F_{Y \rightarrow X}$ where $X$ is a ROI in the left hemisphere and $Y$ the homologous ROI in the right hemisphere. The bidirectional information flow was higher in controls than patients (Fig. 3c) (Two-way ANOVA on bidirectional DC with group and network as factors: effect of group, $F(1,137)=4.0, p=0.04)$. Furthermore, both patients and controls presented a homotopic DC asymmetry (Fig. 3f). In patients, the flow was preferentially from the healthy to the lesioned hemisphere. In healthy participants, the flow was preferentially from the left (dominant) towards the right (non-dominant) hemisphere. The asymmetry was more consistent in patients, who had a higher effect than controls especially in VIS and AUD networks (Two-way ANOVA on bidirectional $D C$ with group and network as factors: effect of group, $F(1,137)$ $=2.0, p=0.2$, group $\times$ network interaction $F(9,137)=2.0, p=0.03$; posthoc T tests patients $v$ s controls for each network, $p<0.05$ in VIS, AUD, FDRcorrected for 10 comparisons).

In summary, patients presented a reduced homotopic information flow, with most of the effect stemming from a reduction of homotopic DC from the lesioned to the healthy hemisphere. Accordingly, stroke patients presented an asymmetric interhemispheric information flow, 
with a stronger flow from the healthy to the lesioned hemisphere. While controls also presented an asymmetric interhemispheric information flow (from the left to the right hemisphere), the asymmetry was weaker than the one observed in patients.

\subsection{Intra-hemispheric undirected functional connectivity and Granger causality analyses}

We then investigated intra-hemispheric UFC, IC, and DC. Previous studies reported a bilateral increase of specific intra-hemispheric UFC links (Baldassarre et al., 2014; Eldaief et al., 2017; Ramsey et al., 2016; Siegel et al., 2016b). As shown in Fig. 4a and b, overall we observed only a weak (nonsignificant) increase of intra-hemispheric UFC in patients (three-way ANOVA with group, network and hemisphere as factors on intrahemispheric UFC; effect of group $F(1,137)=3.1, p=0.07$ ). However, the UFC was higher in the healthy hemisphere than the lesioned one for patients (effect of hemisphere $F(1,2603)=12.2, p=5 \cdot 10^{-4}$; group $\times$ hemisphere interaction $F(9,2584)=10.8, p=0.001$ ), such that patients presented a higher UFC in the healthy hemisphere compared to controls. The effect was concentrated in the CON, VAN, DAN, DMN (network $\times$ hemisphere interaction $F(9,2584)=5.1, p=5 \cdot 10^{-7}$, network $\times$ hemisphere $\times$ group interaction $F(9,2584)=2.6, p=0.004$; post-hoc T tests patients $v$ s controls on healthy hemisphere UFC for each network, $p<0.05$ in CON, $D A N, V A N, D M N, F D R$-corrected for 10 comparisons). Thus, if we define an imbalance in intra-hemispheric UFC as the difference in intrahemispheric UFC between the healthy and the lesioned hemisphere (left and right for controls), patients, but not controls, presented an imbalance (Fig. 4c). The imbalance was highest in AUD, CON, SUB (twoway ANOVA on intra-hemispheric UFC imbalance with network and group as factors, effect of group: $F(1,137)=4.45, p=0.04$; network $\times$ group interaction $F(9,137)=4.0, p=4 \cdot 10^{-5}$; post-hoc $T$ tests patients vs controls on intrahemispheric UFC imbalance for each network, $p<0.05$ in AUD,

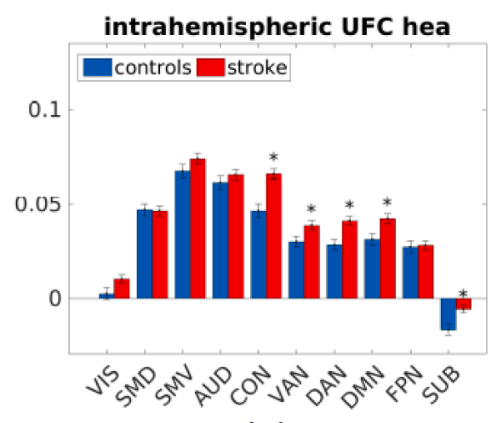

(a)

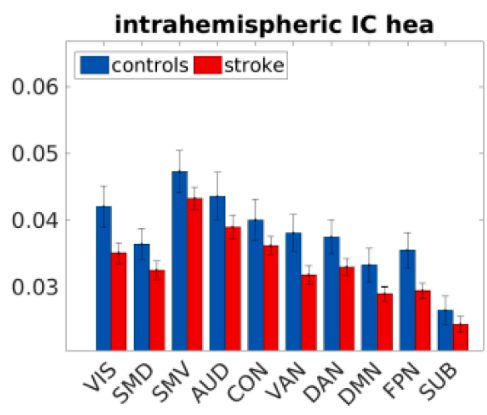

(d)

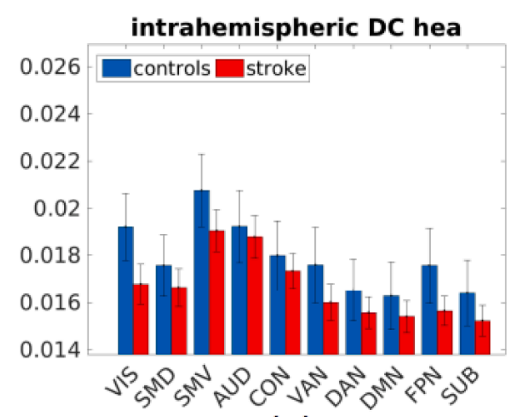

(g)

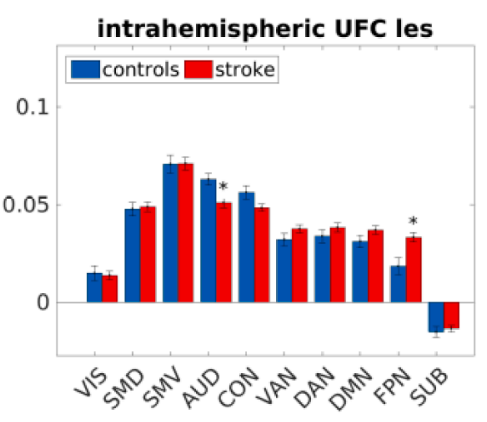

(b)

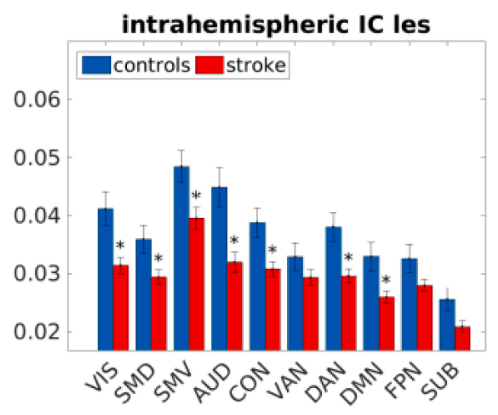

(e)

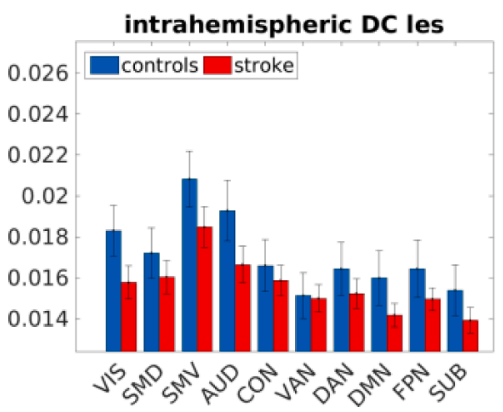

(h)

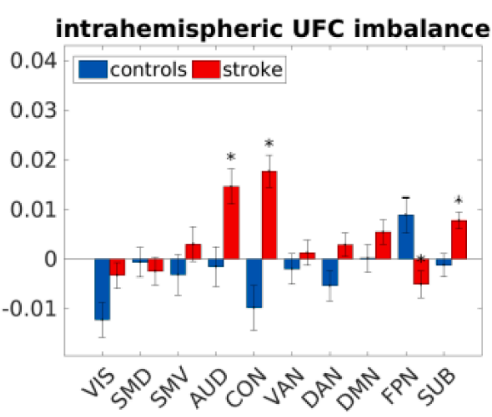

(c)

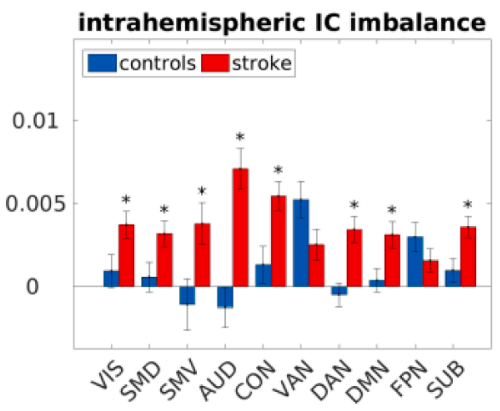

(f)

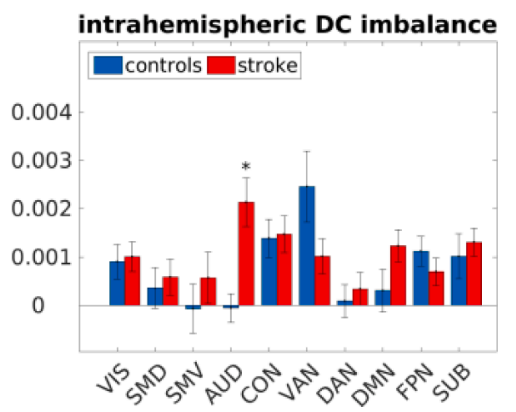

(j)

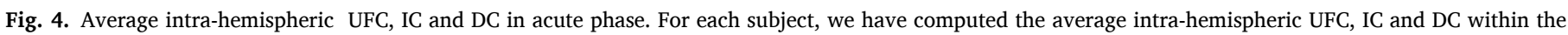

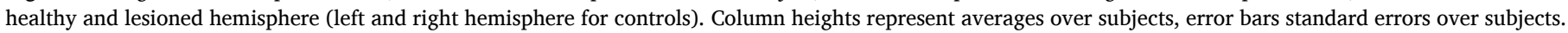

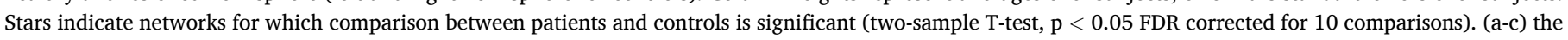

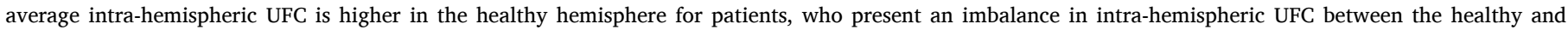

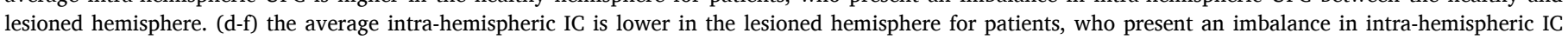

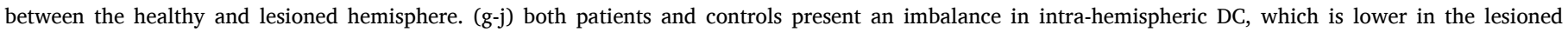
(right) hemisphere. 
CON, SUB, FDR-corrected for 10 comparisons).

The average intra-hemispheric IC was reduced in patients as compared to controls (three-way ANOVA with group, network and hemisphere as factors on intrahemispheric IC; effect of group $F(1,137)=5.1, p=$ 0.02). Much of the effect comes from a reduction of IC in the lesioned hemisphere in patients (effect of hemisphere $F(1,2603)=116.2, p<$ $10^{-10}$, group $\times$ hemisphere interaction $\left.F(9,2584)=13.2, p=2 \cdot 10^{-4}\right)$. The effect was present in most networks (post-hoc T tests controls vs patients on lesioned intra-hemispheric IC, $p<0.05$ in all networks except VAN, FPN, $S U B)$. Thus, if we define an imbalance in intra-hemispheric IC as the difference in intra-hemispheric IC between the healthy and the lesioned hemisphere (left and right for controls), patients, but not controls, presented an imbalance (Fig. 4f). The imbalance was significant in most networks (two-way ANOVA on intra-hemispheric IC imbalance with network and group as factors, effect of group: $F(1,137)=4.45, p=0.04$; network $\times$ group interaction $F(9,137)=4.0, p=4 \cdot 10^{-5}$; post-hoc T tests patients $v$ controls on intrahemispheric IC imbalance for each network, $p<$ 0.05 in all networks except VAN, FPN, SUB, FDR-corrected for 10 comparisons).

Finally, we analyzed DC within each hemisphere. The average intrahemispheric DC was not significantly changed in patients as compared to controls (three-way ANOVA with group, network and hemisphere as factors on intrahemispheric $D C$; effect of group $F(1,137)=0.8, p=0.37$ ). Both patients and controls presented a difference between hemispheres (effect of hemisphere $F(1,2603)=116.2, p<10^{-10}$, group $\times$ hemisphere interaction $F(9,2584)=0.8, p=0.37$ ), with a higher DC in the healthy hemisphere compared to the lesioned one (left and right for controls). Fig. $4 \mathbf{j}$ shows the distribution of the intra-hemispheric DC imbalance (difference between the average intra-hemispheric DC in the healthy and lesioned hemisphere) for patients and controls. Both patients and controls present an imbalance.

\subsection{Differences between patients with left and right lesions}

In the preceding section, we identified a reduction in the strength of inter-hemispheric coupling, as well as imbalances between the healthy and lesioned hemisphere for patients. We now focus with more detail on the group of patients, analyzing possible differences in the strength of the effects between patients with $\mathrm{LH}$ and RH lesions (Fig. 5). When comparing homotopic information flow, effect sizes were comparable for $\mathrm{LH}$ and $\mathrm{RH}$ patients. The two groups presented comparable values of homotopic UFC, IC, and bidirectional DC (Two-way ANOVA with groups and network as factors on homotopic UFC: group $F(1,111)=0.8, p=0.37$, group $\times$ network interaction $F(9,111)=1.2, p=0.28$; Two-way ANOVA with groups and network as factors on homotopic IC: group $F(1,111)=1.3$, $p=0.26$, group $\times$ network interaction $F(9,111)=2.0, p=0.04$; Two-way ANOVA with groups and network as factors on bidirectional homotopic DC: group $F(1,111)=3.1, p=0.08$, group $\times$ network interaction $F(9,111)=$ $1.5, p=0.13)$. When comparing inter-hemispheric imbalances, we observed a tendency for imbalances to be stronger for RH patients. The homotopic DC asymmetry was marginally higher in RH than LH patients (Two-way ANOVA with groups and network as factors on homotopic DC asymmetry: group $F(1,111)=3.3, p=0.07$, group $\times$ network interaction $F$ $(9,111)=1.2, p=0.28)$. The intra-hemispheric IC imbalance was significantly stronger in $\mathrm{RH}$ than LH patients (Two-way ANOVA with groups and network as factors on intrahemispheric IC imbalance: group $F$ $(1,111)=9.4, p=0.002$, group $\times$ network interaction $F(9,111)=3.3, p=$ 0.0005). The intrahemispheric DC imbalance was comparable for the two groups (Two-way ANOVA with groups and network as factors on intrahemispheric DC imbalance: group $F(1,111)=0.4, p=0.49$, group $\times$ network interaction $F(9,111)=2.2, p=0.02)$.

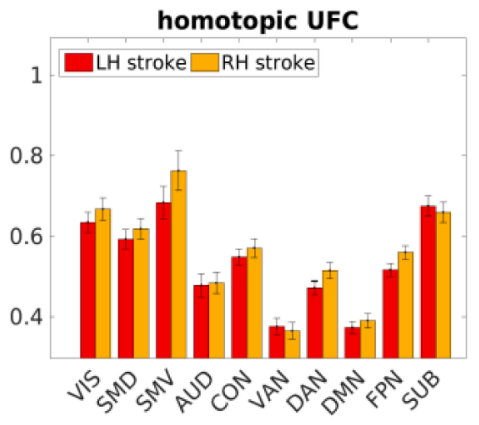

(a)

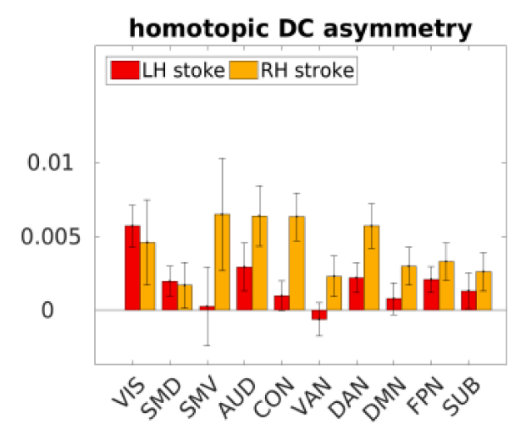

(d)

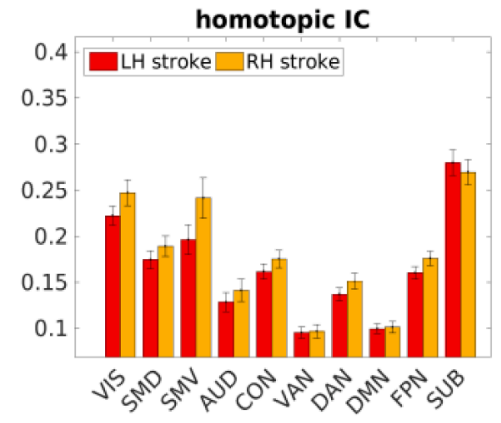

(b)

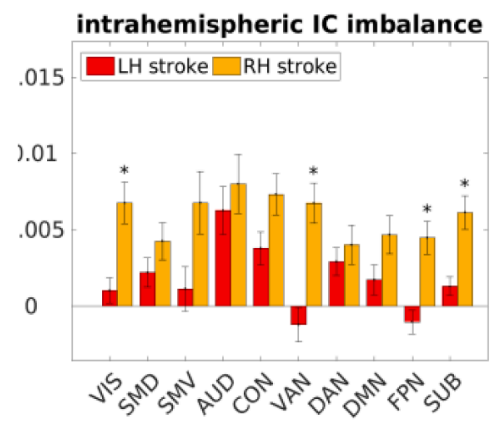

(e)

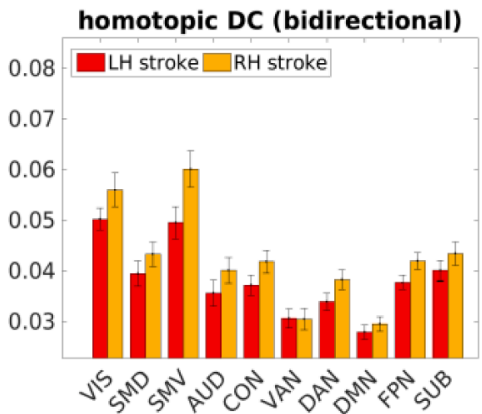

(c)

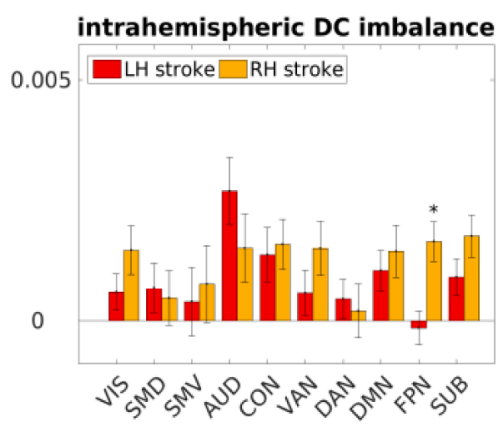

(f)

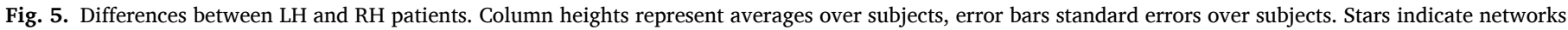

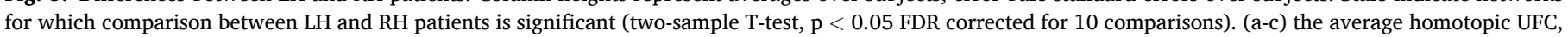

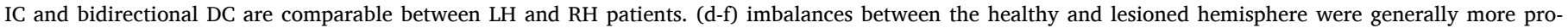
nounced for RH patients than $\mathrm{LH}$ patients. 


\subsection{Global FC and GC summary measures and stroke-related behavioral deficits}

To correlate functional effects with behavioral deficits, we will use summary measures capturing the main effects identified in the preceding analysis of functional connectivity and Granger causality in stroke patients. Each summary measure will be computed at an individual level, and correlated with behavioral scores.

Three summary measures are related to the total level of homotopic connectivity: 1) UFC $_{\text {homo: }}$ average homotopic UFC over all networks; 2) $\mathrm{IC}_{\text {homo: }}$ : average homotopic IC over all networks; 3 ) $\Sigma \mathrm{DC}_{\text {homo: }}$ average bidirectional homotopic DC (contralesional to ipsilesional plus ipsilesional to contralesional) over all networks. Patients, as a group, presented a reduced $\mathrm{UFC}_{\text {homo, }} \mathrm{IC}_{\text {homo }}$ and $\Sigma \mathrm{DC}_{\text {homo }}$. Three additional measures capture imbalances between the lesioned and the healthy hemisphere: 4) $\Delta \mathrm{DC}_{\text {homo: }}$ average homotopic DC asymmetry (lesioned to healthy minus healthy to lesioned) over all networks; 5) $\Delta \mathrm{IC}_{\text {intra: }}$ : average imbalance in intra-hemispheric IC (healthy minus lesioned) over all networks; 6) $\Delta \mathrm{DC}_{\text {intra }}:$ : average imbalance in intra-hemispheric
DC (healthy minus lesioned). Patients present an enhanced $\Delta \mathrm{DC}_{\mathrm{homo}}$ and $\Delta \mathrm{IC}_{\text {intra }}$ in comparison to healthy subjects.

In order to study whether these global summary measures were correlated among each other, we computed the partial Spearman correlation values between pairs of measures for all patients, controlling for lesion volume. Results are shown in Fig. 6a. We observed that the measures split into three groups. The first group included $\mathrm{UFC}_{\text {homo, }}$ $\mathrm{IC}_{\text {homo }}$ and $\Sigma \mathrm{DC} \mathrm{C}_{\text {homo, }}$, which were all strongly correlated. These three measures quantified the strength of inter-hemispheric (homotopic) connectivity. The second group included $\Delta \mathrm{DC}_{\text {homo }}, \Delta \mathrm{IC}_{\text {intra }}$ and $\Delta \mathrm{DC}_{\text {intra, }}$, which were mutually correlated and uncorrelated with the homotopic measures. These three measures quantified homotopic imbalance. A PCA with oblique factors on the (z-scored) summary measures identified two principal components explaining $37 \%$ and $33 \%$ of the total variance respectively (Fig. 6b), henceforth indicated as the principal components PC1 and PC2. The PC1 loaded on $\mathrm{UFC}_{\text {homo }}, \mathrm{IC}_{\text {homo }}$ and $\Sigma \mathrm{DC} \mathrm{C}_{\text {homo, }}$, whereas the $\mathrm{PC} 2$ on $\Delta \mathrm{DC}_{\mathrm{homo}}, \Delta \mathrm{IC}_{\text {intra }}$ and $\Delta \mathrm{DC}_{\text {intra. }}$ Intuitively, $\mathrm{PC} 1$ summarized the inter-hemispheric functional integration (Fig. 6b, left panel), whereas PC2 the inter-hemispheric imbalance (Fig. 6b, right panel). We

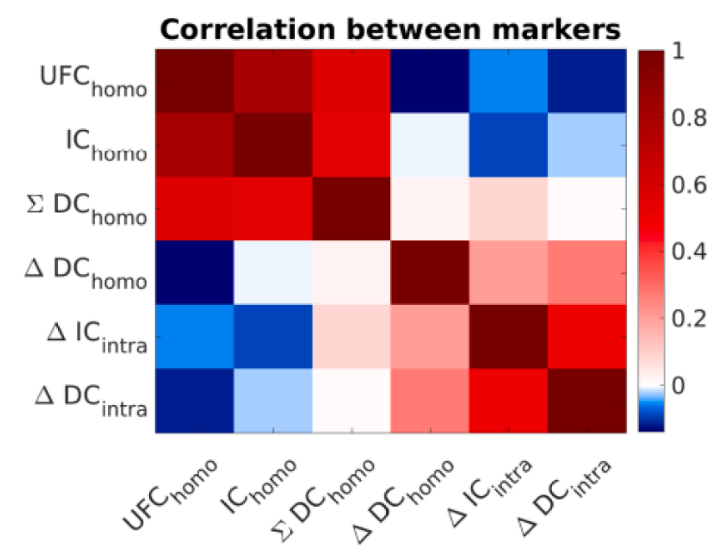

(a)

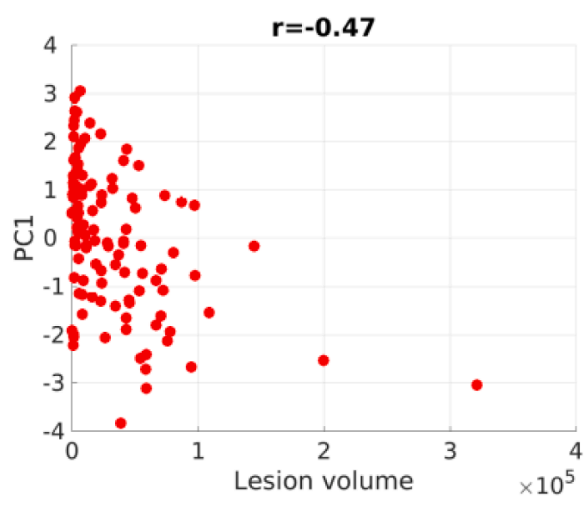

(c)

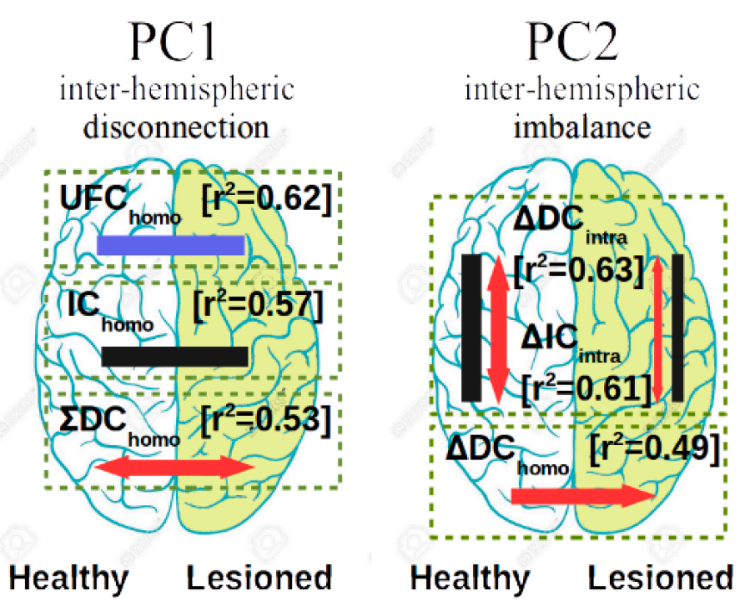

(b)

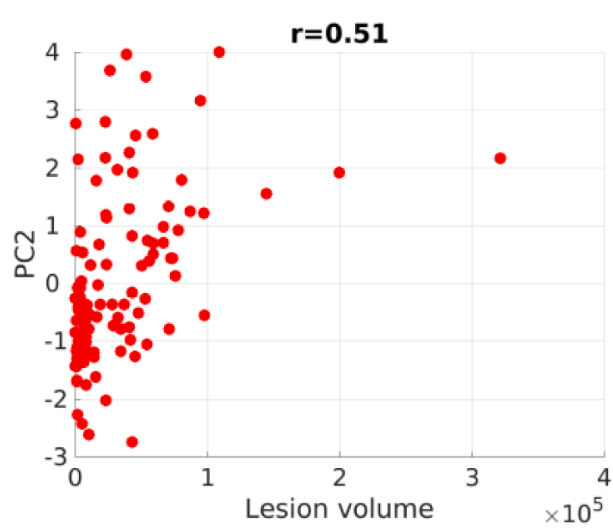

(d)

Fig. 6. Global FC and GC summary measures. Our analysis identified several global correlates of stroke, or summary measures, based on functional connectivity and Granger causality analyses. (a) Looking at the correlation (partial Spearman correlation correcting for lesion volume) between each pair of measures, one can immediately notice two separate groups of correlated measures, one including $\mathrm{UFC}_{\text {homo }}$, $\mathrm{IC}_{\text {homo }}, \Sigma \mathrm{DC}_{\text {homo }}$, the other including $\Delta \mathrm{DC} \mathrm{C}_{\text {homo, }} \Delta \mathrm{IC}$ intra, $\Delta \mathrm{DC}$ intra. $(\mathrm{b}) \mathrm{A} \mathrm{PCA}$ on the seven measures revealed two PCs explaining $>32 \%$ and $30 \%$ of the total variance across patients. The first component (PC1) loaded on UFC homo $_{\text {, IC }}$ homo, $\Sigma \mathrm{DC}_{\text {homo, }}$, the second component (PC2) on $\Delta \mathrm{DC}_{\mathrm{homo}}, \Delta \mathrm{IC}_{\text {intra }}, \Delta \mathrm{DC}$ intra. This is summarized in the two brain plots showing intuitively the main effects captured by $\mathrm{PC} 1$ and PC2 in the healthy and lesioned hemisphere. (c) PC1 correlates negatively with lesion volume (d) the modulus of PC2 correlates positively with lesion volume. 
investigated whether PC1 and PC2 were related to the structural lesions. As shown in Fig. 6c, PC1 was negatively correlated with lesion volume (Spearman $r=-0.47, p\left\langle 10^{-6}\right.$ ): the larger the lesion, the lower the functional integration between the hemispheres (Fig. 6c). Concerning PC2, we found that the modulus of PC2 was positively correlated with lesion volume (Spearman $r=0.51, p\left\langle 10^{-6}\right.$ ): the larger the lesion, the larger the asymmetry between the hemispheres (Fig. $6 \mathrm{~d}$ ). In this regard, we should note that the value PC2 reflected the direction of the asymmetry (left-ward or right-ward), while its modulus reflected the magnitude of the asymmetry.

In a previous work (Corbetta et al., 2015), eight behavioral scores were identified, corresponding to the eight strongest principal components explaining a large fraction of variance in behavioral tests covering language, memory, motion and attention function. The eight factors were associated with language, left body motion, right body motion, spatial attention (hemispatial neglect), sustained attention, shifting attention, spatial memory, verbal memory. Higher scores signify better performance. Right body motion, language, verbal memory and shifting attention scores tend to be lower for LH patients, sustained attention scores show no hemispheric bias, while left body motion and spatial memory scores tend to be lower for RH patients.
We then quantified to which extent the first two principal components (PC1 and PC2) were predictive of the observed behavioral deficits. To do so, we computed the Spearman correlation between the two PCs and behavioral scores (Fig. 7a). We had two general predictions. First, we expected a positive correlation between performance and interhemispheric integration (Carter 2010, Siegel 2016, Corbetta 2018). Consequently, we expected PC1 to correlate positively with behavioral scores. Second, we expected that a decrease of connectivity within and from the lesioned hemisphere would have been generally detrimental for performance. Hence, behavioral scores were expected to correlate negatively with PC2. These expectations were partially met. PC1 correlated positively with all scores for both LH and RH patients (LHP: Spearman $r>0.30$ for all scores except Mot L, Sp Att; RHP: Spearman $r>$ 0.20 for all scores except Shift Att). It is noteworthy that different correlations were significant in LH and RH patients. For LH patients, effects were stronger for scores related to verbal function, general attention, and contralesional motion $(r>0.30, p<0.05$ for Lang, Ver Mem, Shift Att, Mot L, FDR-corrected for 8 comparisons). For RH patients, effects were stronger for scores related to spatial processing and motion $(r>0.45, p$ $<0.05$ for Sp Mem, Sp Att, Mot L, Mot R, FDR-corrected for 8 comparisons). PC2 correlated negatively for LH patients (Spearman $r<-0.17, p$

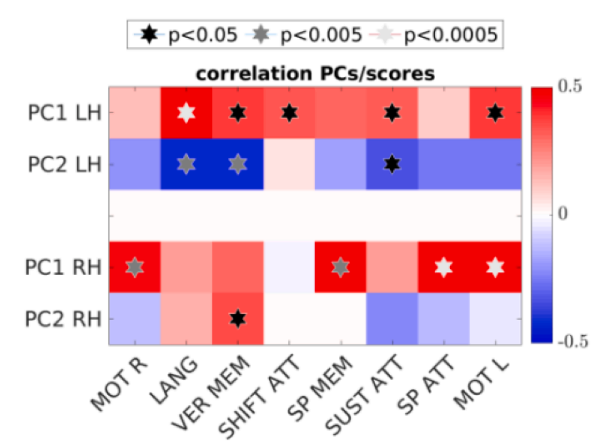

(a)

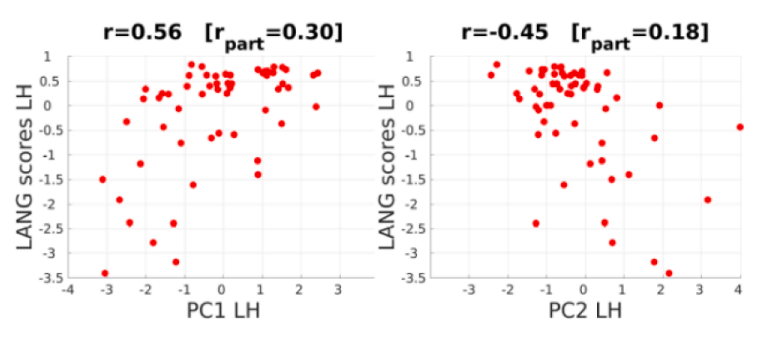

(c)

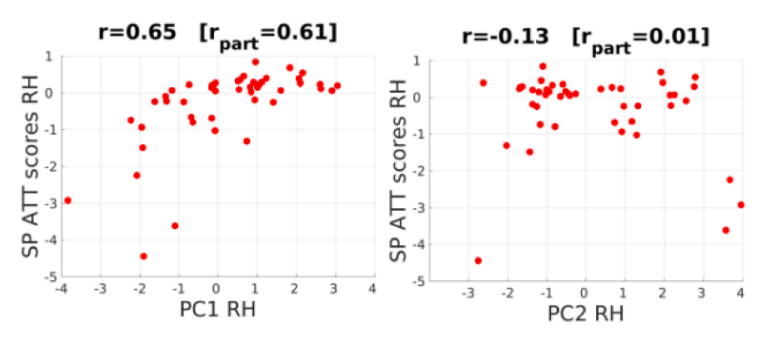

(e)

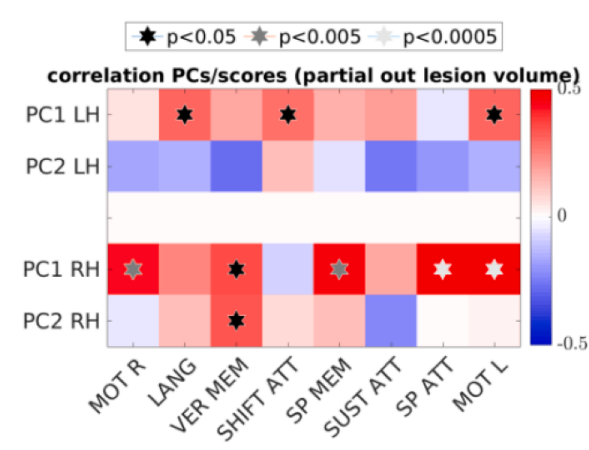

(b)
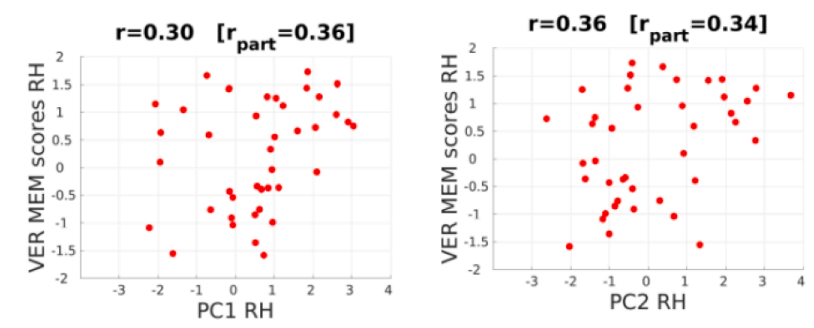

(d)
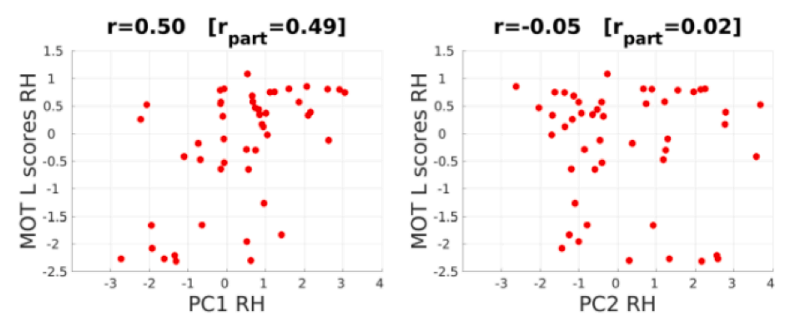

(f)

Fig. 7. Correlation with behavioral scores. (a) Spearman correlation between behavioral scores and the two principal components (PC) summarizing FC and GC stroke summary measures (b) partial Spearman correlation between behavioral scores and the two principal components, correcting for lesion volume (c) scatter plot of PC1/PC2 versus language scores for LH patients (d) scatter plot of PC1/PC2 vs verbal memory scores for RH patients (e) scatter plot of PC1/PC2 vs spatial attention scores for RH patients (f) scatter plot of PC1/PC2 versus left body motion scores for RH patients. 
$<0.27$ uncorrected for all scores except Shift Att), and was significant for scores associated with language $(r<-0.43, p<0.05$ for Lang, Ver Mem, FDR-corrected for 8 comparisons), while correlations were weaker, ambiguous in sign, and not significant for RH patients ( $p>0.05$ for all scores, FDR-corrected for 8 comparisons); the strongest observed effect was a positive correlation between PC2 and language-related scores $(r=$ $0.16, p=0.26$ for Lang; $r=0.36, p=0.02$ uncorrected for Ver mem). In summary, a lower PC1 (lower inter-hemispheric integration) was associated with significantly worse performance in several behavioral domains, different for LH and RH patients. A higher PC2 (higher interhemispheric imbalance) was associated with worse performance in language-related domains for LH patients, and with better performance in $\mathrm{RH}$ patients.

\subsection{Control analyses}

Part of the observed differences between LH and RH patients may be due to the influence of lesion volume. Both the homotopic UFC and the homotopic IC appeared to be higher for RH than LH patients (Fig. 3). In both cases the difference could be largely explained by differences in lesion volume (UFC: one-way ANOVA with group as factor, after linearly regressing logarithm of lesion volume lesion: $F(1,1110)=1.5, p=0.2 ;$ IC: one-way ANOVA with group as factor, after linearly regressing logarithm of lesion volume lesion: $F(1,1110)=3.4, p=0.06)$. Moreover, since PC1 and PC2 correlate with lesion volume, part of the observed correlation with behavioral scores may be explained by lesion volume. A larger lesion volume is causally related to more widespread structural disconnections (Griffis et al., 2019), which are at the root of functional connectivity alterations captured by PC1, PC2. However, a larger lesion volume is also causally associated with a larger local damage to cortical area. Thus, a correlation between the PCs and behavioral scores does not provide sufficient evidence that the functional anomalies captured by the PCs have a specific role in the genesis of the deficits, more than other functional perturbations such as impaired activity in the locally damaged area. We computed the partial Spearman correlation between the two PCs and behavioral scores, controlling for the effect of lesion volume (Fig. 7b). As for PC1, we still observed a positive correlation with behavioral scores (LHP: Spearman $r>0.13$

for all scores except Sp Att; RHP: $r>0.26$, for all scores except Shift Att). Surprisingly, while effects were generally reduced and no longer significant for LH patients ( $p>0.05$ for all scores, FDR-corrected for 8 comparisons), correlations remained significant for RH patients $(r>$ 0.36, $p<0.05$ Mot R, Sp Mem, Sp Att, Mot L, FDR-corrected for 8 comparisons). For PC2, we still observed a generally negative correlation for LH patients (Spearman $r<-0.08$, for all scores except Shift Att), but correlations were no longer significant ( $p>0.05$ for all scores, FDRcorrected for 8 comparisons). Correlations were still not significant for RH patients ( $p>0.05$ for all scores, FDR-corrected for 10 comparisons).

We finally performed control analyses to investigate potential confounding effects associated with nuisance sources and hemodynamic lags. GC analyses were performed on preprocessed BOLD signals without global signal regression (GSR) removal. The rationale for this choice was that GSR may effectively work as a "temporal filter" (Liu et al., 2017), suppressing the contribution of time points associated with low global signal, potentially distorting the estimation of information flows in GC. While standardly adopted for UFC estimation, GSR is a contentious step (Saad et al., 2012), particularly when one compares healthy subjects with neurological or psychiatric patients (Hahamy et al., 2014; Yang et al., 2014). Indeed, the global signal can reflect extended correlation of neural origin (Scholvinck et al., 2010), possibly differing between patients and control subjects. By applying GSR to our data, homotopic information transfer (homotopic IC and bidirectional DC) presented similar effects to those found without GSR, including the asymmetry in homotopic DC (Fig. 8a). However, results on intra-hemispheric GC differed: no clear imbalance was observed in intra-hemispheric DC or IC. Thus, GSR significantly attenuates the hemispheric imbalances.
However, due to the high network specificity of the observed imbalances, it appears unlikely that such imbalances represent metabolic, movement, breathing-rate, cardiovascular or vigilance effects. It is more likely that differences in global signal between the hemispheres represent alterations in the excitation/inhibition balance within each hemisphere (Yang et al., 2014), which are obscured by GSR.

Hemodynamic lags represent an additional potential confound for our results. In fact, stroke can cause a pathologic delay in the hemodynamic response in the perilesional area, or in a wider area subserved by the occluded artery (Siegel et al., 2016b). This delay may introduce spurious "lags" of non-neural origin between regions in this area and homologous regions in the intact hemisphere, thus contributing to the observed homotopic DC asymmetry. We checked whether the observed global homotopic DC asymmetry could be linked to asymmetries in the perilesional area. We considered each region $X$ in the lesioned hemisphere and computed the DC asymmetry $G_{Y \rightarrow X}=F_{Y \rightarrow X}-F_{X \rightarrow Y}$ where $Y$ is the homologous area in the intact hemisphere. We thus obtained brainwide maps of homotopic DC asymmetry that overlayed with the lesion maps (to produce the homotopic DC maps, we assigned the value $G_{Y \rightarrow X}$ to all voxels within a radius of $10 \mathrm{~mm}$ around the center of each ROI $X$, and then applied $10 \mathrm{~mm}$ Gaussian smoothing). In Fig. 8d, we show the results for a representative subject. The strongest DC asymmetries were observed far from the lesions location in the brain. In order to have a more quantitative control, we repeated our analyses excluding all regions at a distance $<4 \mathrm{~cm}$ from the lesioned area. As shown in Fig. $8 \mathrm{~b}$, the homotopic DC asymmetry is still present after this removal, while the intra-hemispheric IC and DC imbalance appear to be even strengthened. This showed that the observed effects are not due to anomalous hemodynamic lags in the vicinity of the lesion.

To further verify whether the asymmetries may be driven by general differences in the hemodynamic response between the healthy and lesioned hemisphere, we estimated the hemodynamic response at each region by the "blind deconvolution" approach described in (Wu et al., 2013), which is the only existing approach to characterize the hemodynamic response in resting state fMRI. Assuming that the neural signal underlying the BOLD is generated by a point process (a rather strong assumption), peaks of the BOLD signal correspond to neural "events" convolved with the hemodynamic response function (HRF), which allows estimating the HRF. This method may be quite inaccurate with our data, given the low temporal resolution at hand $(T R=2)$. Despite its limitations, the method may capture large differences in the shape of between the healthy and lesioned hemisphere. We used the software provided by the authors of the approach (https://pypi. org/project/rsHRF/, with default parameters, using the gamma estimation method, and using Wiener deconvolution. For each region, we extracted the HRF, whose shape can be summarized by 3 parameters corresponding to the amplitude, time-to-peak and FWHM of each region's HRF (Wu et al. 2013). Larger time-to-peak and FWHM correspond to slower responses. For each subject, we computed the average HRF amplitude, time-to-peak and FWHM in the healthy and lesioned hemisphere separately, and considered the difference, obtaining indexes of hemispheric imbalances in the HRF amplitude ( $\Delta \mathrm{amp})$, time-to-peak $(\Delta \mathrm{TTP})$ and FWHM $(\Delta \mathrm{FWHM})$. In Fig. $3 \mathrm{c}$, we show the average HRF imbalances for patients. We did not observe significant global HRF imbalances. In Fig. 8e, we overlay the homotopic DC asymmetry map with a map of the homotopic imbalance in HRF parameters (for each region, we computed the difference in amplitude, time-to-peak and FWHM asymmetry with the corresponding homologous region). The strongest DC asymmetries were not in close correspondence with the homotopic asymmetries in the FWHM of the HRF (the parameter most likely associated to longer hemodynamic responses).

\section{Discussion}

Previous research, including previous work on the Washington stroke database (Siegel et al., 2016a, Corbetta et al., 2018, Griffis et al. 


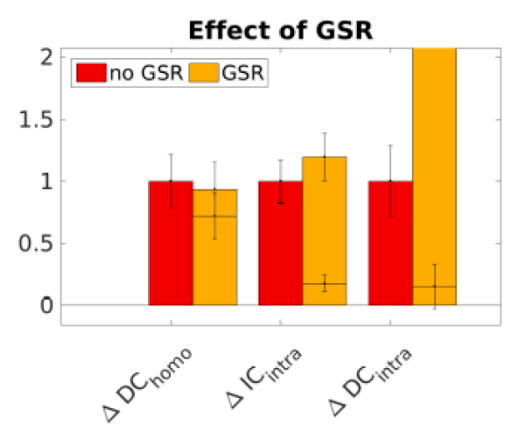

(a)

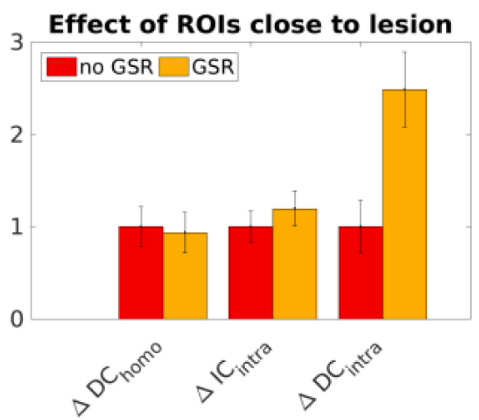

(b)

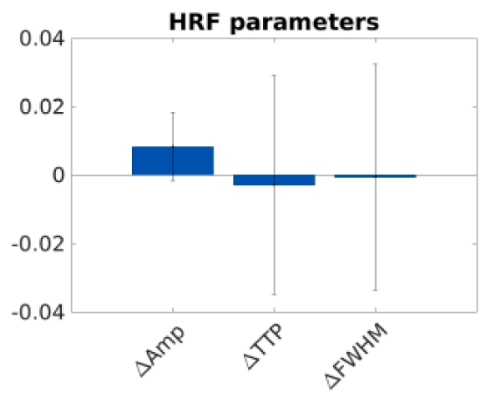

(c)

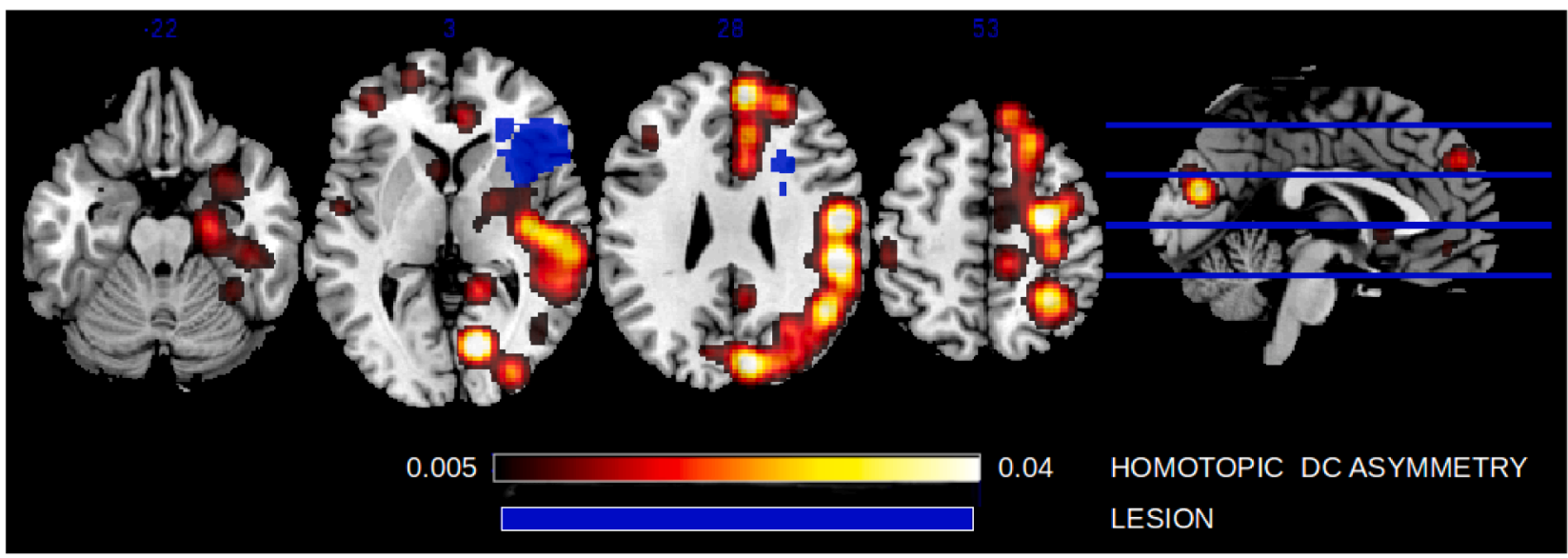

(d)

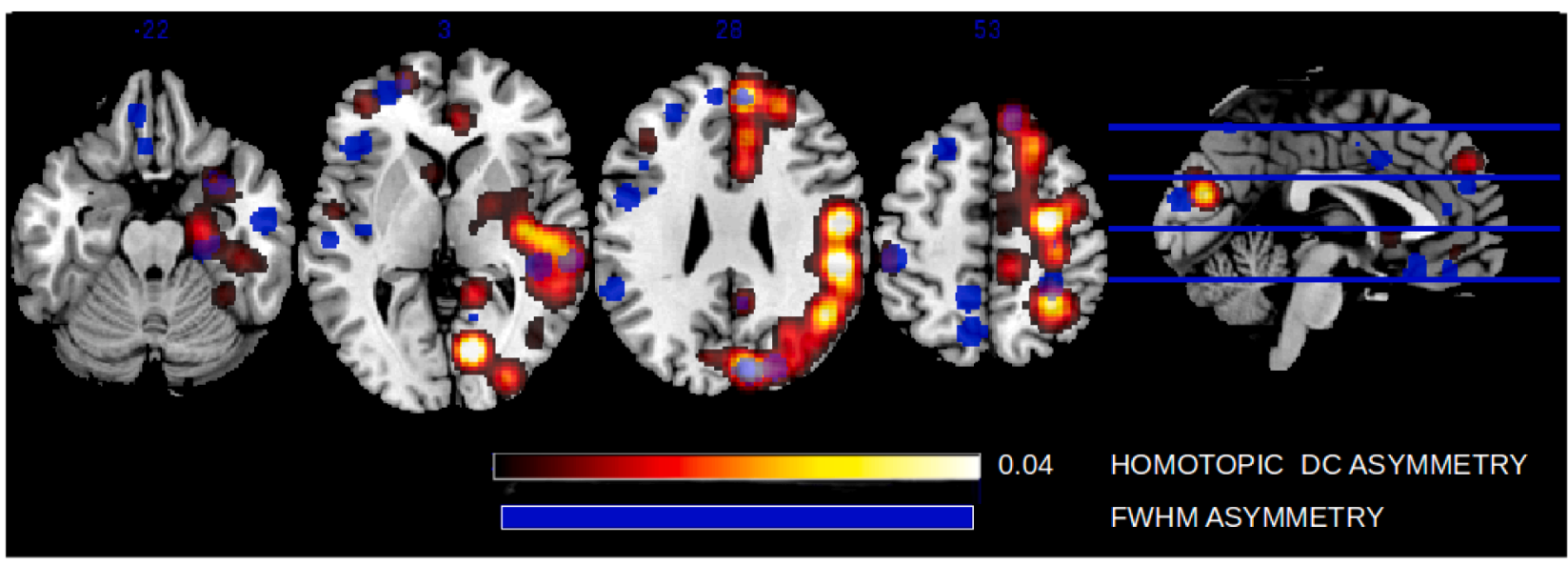

(e)

Fig. 8. Control for possible confounds. (a) We checked the effect of GSR on the found inter-hemispheric imbalances. We show the average imbalances in homotopic DC, intra-hemispheric IC, and intra-hemispheric DC before and after GSR (for each column, results have been rescaled to the average results without GSR for visualization purposes). GSR has no effect on the homotopic DC asymmetry, while it removes the imbalance in intra-hemispheric IC and DC. (b) We show the average imbalances in homotopic DC, intra-hemispheric IC, and intra-hemispheric DC with or without removing from analysis all regions at a distance $<4 \mathrm{~cm}$ form the lesion (for each column, results have been rescaled to the average results without GSR for visualization purposes). Such removal has no effect on the homotopic DC asymmetry, while it strengthens the imbalance observed in intra-hemispheric IC and DC. (c) We show the average hemispheric asymmetry in the estimated HRF parameters. Patients did not present significant asymmetries in HRF between the healthy and the lesioned hemisphere (d) We show a map of the homotopic DC asymmetry for one representative subject, together with the lesion location (in blue). Strongest homotopic DC asymmetries are found far from the lesion (e) We show a map of the homotopic DC asymmetry for the same subject as in (d), together with the asymmetry in the FWHM of the estimated hemodynamic response. Strongest homotopic DC asymmetries are not in correspondence with asymmetries in the FWHM of the hemodynamic response. (For interpretation of the references to colour in this figure legend, the reader is referred to the web version of this article.) 
2019), has made extensive use of resting-state fMRI to investigate functional connectivity in stroke patients. The results of these studies suggest a view of stroke as a network dysfunction syndrome. Stroke is accompanied by widespread alterations of functional connectivity, with common patterns observed across patients independently of lesion location. In particular, most patients present a loss of inter-hemispheric FC (Corbetta et al., 2018; Golestani et al., 2013; Siegel et al., 2016a; Tang et al., 2016). Anomalies of long-range FC are paralleled by perturbations of monosynaptic (Griffis et al. 2019) and polysynaptic (Griffis et al. 2020) structural connections. While sensorimotor deficits are reasonably well explained by local damage, cognitive deficits are better explained by network dysfunction (Siegel et al., 2016b).

However, it was still unclear whether stroke produces functional asymmetries in long-range brain interactions. Being symmetric, standard functional connectivity (UFC) and structural connectivity (SC) measures are not suited to address this question. In our work, we used Granger causality (GC) measures to investigate alterations of long-range directional interactions in the brain after stroke. By exhaustively looking at temporal dependencies between the BOLD signals of two regions, GC measures can yield information about the directionality and time scale of interactions, which is missing from UFC analyses. Our analyses revealed several stroke-related asymmetries between the hemispheres, which further allowed us to better highlight major differences between patients with left- or right-hemisphere lesions which had not been specifically addressed in previous analyses.

\subsection{Stroke-related modulations in inter- and intra-hemispheric coupling revealed by Granger causality analyses}

One of the major functional effects of stroke is a loss of interhemispheric integration associated with a decrease of homotopic UFC. It is still relatively unknown to which extent the UFC decrease corresponds to a decrease of direct interactions (supported by homotopic connections crossing the corpus callosum (Schmahmann et al., 2009)) or indirect interactions through subcortical structures. Our results from GC-based analyses show that UFC decrease (Fig. 3a) is strongly associated with a loss of inter-hemispheric interactions captured by the homotopic IC and DC (Fig. 3b and c). IC captured cortico-cortical interactions unfolding within $1 \mathrm{TR}$, while DC captured lagged corticocortical interactions occurring on a time scale longer than 1 TR. Classically, IC are interpreted as originating from external common inputs (Ding et al., 2006). Since a large part of the total interdependence between the signals of homotopic areas is due to the IC (Fig. 2c), our results suggest that a component of stroke-related alterations in cortico-cortical coupling emerges from disrupted common inputs from regions that project symmetrically to cortical areas, such as subcortical structures. This hypothesis is supported by structural analyses that locate stroke lesions primarily in subcortical areas, such as the thalamus (Corbetta et al. 2015), as well as by recent experimental work showing that subcortical structures can play a large role in maintaining FC between cortical regions when direct influences are impaired (Canella et al. 2020). However, given the slow sampling rate of our data (TR $=2 \mathrm{~s}$ ), an IC decrease cannot be uniquely attributed to a loss of common input, as it may also result from a decrease of fast directed interactions occurring on timescale shorter than $2 \mathrm{~s}$. To which extent subcortical structures contribute in re-modulating cortical interaction remains a relevant topic for further investigation.

Importantly, even though IC rather than DC dominates homotopic interdependence, DC analysis is precious as it hints at strong interhemispheric communication asymmetries. Our results on homotopic DC showed that stroke impacts the inter-hemispheric information flow asymmetrically, with a spared information flow from the healthy to the lesioned hemisphere and a reduced flow in the opposite direction (Fig. 3d-f). This asymmetric effect is not immediately explained by structural lesions, since there is no evidence that ischemia would affect selectively fibers from the ipsilesional to the contralesional hemisphere rather than in the opposite direction. The homotopic asymmetry we measured is in line with recent work (Wang et al., 2019) showing that time series in the lesioned hemisphere are "lagged" with respect to the homologous areas in the healthy hemisphere. To which extent this effect may stem from non-neural, hemodynamic causes - a systematic alteration of the hemodynamic response in the lesioned hemisphere - remains an open question. In our control analysis (Fig. 8) we excluded the possibility that the effect be trivially related to the well-known presence of large hemodynamic lags in the perilesional area (Siegel et al., 2016b). Thus, the measured asymmetry, if caused by hemodynamic effects, would imply wide alterations of the hemodynamic response far from the lesion. In future work, this issue may be specifically addressed by applying deconvolution prior to GC analysis, building on "blind deconvolution" techniques that allow retrieving the hemodynamic response from resting-state data (Wu et al., 2013). Such analysis is beyond the scope of the current study. Since deconvolution techniques for resting-state fMRI remain exploratory, it is still unclear whether these methods are accurate in presence of anomalous distortions of the hemodynamic response potentially arising in pathological conditions such as stroke.

The relevance of the observed homotopic GC asymmetry is strengthened by our analysis of intra-hemispheric GC, which revealed another functional imbalance between the hemispheres in stroke patients: intra-hemispheric IC and DC are higher in the intact hemisphere than the lesioned one (Fig. $4 \mathrm{f}$ and $\mathrm{j}$ ). Our results are not conclusive regarding the relation between the homotopic DC asymmetry (Fig. 3f) and the imbalance in intra-hemispheric IC and DC (Fig. 4f and j). However, we provided evidence that the intra-hemispheric and interhemispheric imbalances are correlated (Fig. 6), which suggests that the two results are not independent and may have a common cause. We speculate that both effects could stem from structural disconnection within the lesioned hemisphere, causing a loss of inter-areal excitatory influences. Since stroke can damage structural connections between ipsilesional areas, we could generally expect a loss of excitatory influences, and hence general activity decrease, within the lesioned hemisphere (Grefkes and Fink, 2014). This, in turn, would also imply that the lesioned hemisphere would exert less excitation on the healthy one. This picture would explain both the decrease of ipsilesional DC and IC, and the decrease of DC from the lesioned to the healthy hemisphere. Further support to this interpretation comes from the fact that all imbalance measures $\left(\Delta \mathrm{DC}_{\text {homo }}, \Delta \mathrm{IC}_{\text {intra }}, \Delta \mathrm{DC}_{\text {intra }}\right)$ correlate negatively with lesion volume (i.e., the stronger the lesion, the higher the intra- and inter-hemispheric functional imbalances).

Post-stroke inter-hemispheric imbalances in effective connectivity were widely reported in the motor system, as reviewed in (Grefkes and Fink, 2014). During motor tasks, excitatory influences within the lesioned hemisphere are reduced, contributing to a general decrease of ipsilesional brain activity (Grefkes and Fink, 2014; Rehme and Grefkes, 2013). As for inter-hemispheric connectivity, several studies on the motor system after stroke indicate an anomalous influence of the contralesional hemisphere onto the lesioned one during motor tasks (Rehme and Grefkes, 2013, Grefkes et al., 2010, Grefkes and Fink, 2014). Whether the contralesional influence is inhibitory (hence detrimental to motor performance), or excitatory (hence supportive of performance) seems to depend on several factors, including time after stroke and severity of the lesions (Pino et al., 2014). Our results instead showed a decrease of influence of the damaged hemisphere on the normal one. However, we are wary of a direct comparison, since our whole-brain results were obtained with a resting-state paradigm, hence without any specific involvement of the motor cortex. In order to further clarify inter-hemispheric balance after stroke, future whole-brain studies should discriminate between excitatory and inhibitory influences, which is not possible in the current GC analysis.

Hemispheric functional imbalance and stroke-related behavioral deficits

Previous behavioral analyses on this cohort (Corbetta et al., 2015; 
Ramsey et al., 2016) identified sets of correlated deficits for left and right lesions respectively, largely agreeing with lateralization maps described in healthy subjects (Karolis et al., 2019). Right body motion, language, verbal memory and shifting attention scores tend to be lower for LH patients, sustained attention scores show no hemispheric bias, while left body motion, spatial attention and spatial memory scores tend to be lower for RH patients. Here, we systematically addressed for the first time the functional basis of $\mathrm{LH} / \mathrm{RH}$ patient differences.

In LH patients, the amount of inter-hemispheric communication (summarized by PC1) correlated positively with behavior for domains that are specific to the left hemisphere (language, verbal memory, attention shifting), with the exception of right motion. The correlation between PC1 and behavioral scores was significantly lower if lesion volume was regressed, and presently we cannot discriminate the specific impact of inter-hemispheric communication loss on behavioral function from other possible effects resulting from the lesion. The imbalance between the hemispheres (summarized by PC2) correlated negatively with behavioral scores, significantly for behaviors that were affected by left hemisphere lesions (language, verbal memory) and could be largely explained by lesion volume, suggesting that it reflects the extent of intrahemispheric LH damage.

In RH patients PC1 correlated with behavior for domains more associated with the right hemisphere (motor function, spatial and sustained attention, and spatial memory). Correlations were robust to regression of lesion volume, which suggests a specific impact of interhemispheric communication loss on behavior. This hypothesis agrees with previous studies showing that deficits that were affected by right lesions were more associated with inter-hemispheric rather than intrahemispheric functional disconnection (Baldassarre et al., 2016a; Baldassarre et al., 2016b; Siegel et al., 2016b). We speculate that input from the LH may be more critical for functional integrity of the RH than the other way around, congruently with studies reporting that the left hemisphere presents more central or indispensable regions for the whole-brain structural network (Iturria-Medina et al., 2011), and that the right hemisphere depends more heavily on integration with the left one than the other way around (Gotts et al., 2013). In RH patients we did not observe a negative correlation between scores and PC2, suggesting that intra-hemispheric damage has a lesser impact on behavior. Instead, we observed a positive correlation between PC2 and verbal memory scores, which suggests a supportive role of the left (contralesional) hemisphere for a left-lateralized function in the case of right lesions.

In both LH and RH patients, sustained attention scores had a significant positive correlation with PC1, and a negative correlation with PC2. This is consistent with previous literature suggesting that higher scores are associated with a higher inter-hemispheric integration and a higher intra-hemispheric integration in the lesioned hemisphere (Corbetta et al. 2005, He et al. 2007, Corbetta and Shulman 2011), but a large part of the correlation observed in this work could be explained by lesion volume, hence at present we cannot know to which extent the effect is causally related to functional connectivity anomalies.

\subsection{Methodological considerations on Granger causality analyses: Advantages and limitations}

The efficacy of bivariate GC as a data-driven analysis method rests on its ability to uncover global patterns of information flow and differences in information flows between groups or experimental conditions in a completely unsupervised way (Faes et al., 2017; Friston et al., 2013; Roebroeck et al., 2011; Roebroeck et al., 2005). Our choice of bivariate GC, instead of multivariate GC (Barnett and Seth, 2014), had several motivations. The first motivation was that we wanted to compare results obtained with a Granger causality approach with previous results obtained with (undirected) functional connectivity. We thus designed the analyses with the aim of using the same 343 ROI atlas used in previous UFC studies. Multivariate approaches present several difficulties when applied to large networks. In standard multivariate analyses, a direct link between two regions is obtained by conditioning on all remaining variables. When the whole network is large, there may be numerical issues, and this is why multivariate GC is usually applied to smaller networks (of the order of 100 nodes) (Tang et al., 2012; Stramaglia et al., 2016). Moreover, even when numerical problems may be bypassed, full conditioning is conceptually problematic when there are subgroups of regions with similar signals (redundancy). In this case, conditioning leads to underestimating true connections (Stramaglia et al. 2014). One solution would be to group regions sharing similar information content before estimating GC, as proposed by Barrett 2010 and Angelini et al. (2010). However, such analysis is still problematic in the current settings, because there currently exist no established and accepted methods to find a good partition of regions into groups for large networks. This is why whole-brain studies commonly use bivariate GC to uncover global patterns of information flow (see e.g., Deco et al. 2021). The second motivation is that our goal was to study network-level FC measures, rather than direct interaction between a specific pair of brain regions. Pair-wise analysis is limited if one wishes to infer the direct interaction between a specific pair of brain regions, but it is not much of a limitation within our analysis, where we always average GC over many pairs of regions to infer network- or even hemisphere-level GC measures, and we are less concerned about the possibility that single connections may include indirect effects. A third motivation to use bivariate GC is its estimability from short signals. This property allows us to estimate GC from BOLD time series of 400-800 time points, i.e., the time series of single subjects. Thus, we do not need to concatenate several subjects to perform the estimation, and we can obtain individual estimates.

The main limitation of our analysis is the uncertainty affecting GC estimates at different levels, from single-session to single-subject (Fig. 2). For each GC-based stroke summary measure (e.g., the total homotopic IC), we obtained a large group variance, and consequently a large overlap between the distributions of patients and controls, so that we could not robustly classify an individual as patient or control based on his/her value of the summary measure. It is likely that part of this variance reflects estimation error, rather than true interindividual variability. Analogously, the uncertainty affecting single-subject estimates also implies a difficulty in relating individual GC results with individual behavioral scores. Thus, estimation error limits the use of GC for the development of personalized biomarkers predictive of clinical condition and behavioral performance at the single-patient level. This limitation is not inherent in GC per se, but depends on the relative paucity of functional data available for each patient, and the poor temporal resolution implied by $\mathrm{TR}=2 \mathrm{~s}$. By taking longer recordings or repeating recording sessions, we could obtain much more accurate GC estimates. Improved GC estimates may also be obtained by using a lower TR. Using a TR $=0.67 \mathrm{~s}$ (as in the Human Connectome Project database (van Essen et al., 2013)) would triple the number of points for estimation and offer a significantly improved time resolution, allowing for a more precise characterization of directionality effects (we predict that by using a shorter TR, a part of the total interdependence that is seen as IC in this study would appear as DC). In our opinion, the main limitation of GC in this study is due to intrinsic properties of the data, rather than the specific approach used for calculating DC - bivariate and covariancebased. We are skeptical that more sophisticated approaches for GC estimation would yield radically improved results. In particular, for several reasons we do not believe that a multivariate approach (Barnett and Seth, 2014), which in principle gives cleaner results by eliminating indirect network effects, would particularly contribute to our study. Since we have a large number of areas, conditioning would impair estimation, especially because of many redundancies (Stramaglia et al. 2016). Moreover, since our main results are based on large averages over many pairs of regions, they are largely indifferent to whether single links are affected by indirect contributions. 


\subsection{Limitations due to possible confounders}

An important limitation of our work is that our analyses are completely based on the BOLD, an indirect measure of neural activity. While the BOLD at rest has a substantial neural component (Scholvinck et al., 2010), the signal is strongly affected by a multitude of confounders, mostly of neurovascular origin.

The first of these confounders is the hemodynamic response function (HRF), which is known to be non-uniform throughout the brain (Aguirre et al., 1998). In principle, one should deconvolve the HRF from the BOLD of each area to obtain the corresponding "neural" time series. However, there is no established method to retrieve the HRF from resting-state data: contrary to task fMRI, where neural activation is timelocked to external events, the underlying neural events are unknown. A possible approach to deal with this issue is to assume that spontaneous events follow a point process (Wu et al., 2013), which allows retrieving the HRF from the large fluctuations of the BOLD. Using this method, we characterized the HRF of each region for patients and controls. A quite surprising result was that patients did not exhibit anomalous or noncanonical HRF shapes. In particular, we might have expected retarded or slowed-down responses in the lesioned hemisphere of patients (Siegel et al 2016b), which may explain part of the observed inter-hemispheric imbalances. This result does not imply that the HRF is normal in patients: quite on the contrary, it may just signify a limited power of blind deconvolution with the limited temporal resolution or our data (TR $=2$ ). To what extent non-uniformities of the HRF throughout the brain may contribute to the observed effects, in particular inter-hemispheric imbalances, thus remains an open question. To better address this issue, we may need to rely on a better acquisition protocol with lower TR.

Another relevant neurovascular confounder comes from inhomogeneities of the blood arrival time in the brain. Erdoğan et al. (2016) argued that the global fMRI signal is to a large proportion due to systemic low frequency oscillations propagating with cerebral blood circulation throughout the brain, and impacting the BOLD signal of different areas with different delays. Then, they proposed to regress a locally time-shifted version of the global fMRI time signal to remove these fluctuations. In future work, advanced methods to correct for time delays such as that proposed by Erdogan et al. could be applied to further clean the signal. This would require significant effort, as possibly complex interactions between anomalies of blood circulation and anomalies of the HRF may emerge, needing a dedicated study.

Finally, another possible confounder is provided by cardiac and respiration effects. An optimal method to correct for cardiac and respiratory effects is to rely on external measurements (Caballero-Gaudes and Reynolds, 2017). Since our acquisition protocol did not include these measurements, this strategy could not be applied to our data.

To date, it is still a matter of investigation how hemodynamic, blood flow, cardiac and respiration artifacts, and the related correction techniques, may impact on GC or effective connectivity estimates. This precludes any quantitative assessment of the role of these artifacts, and correction techniques on FC/GC analyses. For this reason, we adopted a standard preprocessing pipeline instead of including advanced preprocessing steps whose validity and effects are yet to be established. Our preprocessing pipeline included a rather aggressive nuisance regression (we regressed the signal of more than twenty many areas including locations in the white matter, ventricles and extra-axial CSF), but it is unclear whether this preprocessing step suffices to eliminate or substantially limit cardiac, respiration and blood flow effects. The possible presence of these artifacts certainly limits the interpretation of the observed differences in FC and GC, which may be significantly impacted by non-neural confounders. We therefore stress that the neurobiological causes of the observed difference in GC/UFC are yet to be clarified, and remain a relevant subject for future work.

\section{Conclusions}

To conclude, the Granger causality (GC) analysis of inter-areal interactions after stroke highlighted two broad pathological features. First, a decrease of homotopic GC, suggesting a large decrease of interhemispheric communication, either direct or mediated by subcortical structures. Second, an inter-hemispheric imbalance, revealed by an asymmetry in homotopic GC, as well as a right-left difference in intrahemispheric GC, suggesting a decrease of communication within and from the lesioned hemisphere. These results show that previously observed FC alterations in stroke are related to broad changes in interareal communication. Furthermore, our analysis confirms and generalizes previous findings about post-stroke inter-hemispheric imbalances in the motor and attention system. The observed GC anomalies highlighted a different impact of lesion on behavior depending on which hemisphere was lesioned. Left-lateralized behavior was strongly affected by loss of intra-hemispheric communication in patients with left hemisphere lesions. Right-lateralized behavior was strongly affected by loss of interhemispheric communication in patients with right hemisphere lesions.

\section{CRediT authorship contribution statement}

Michele Allegra: Conceptualization, Investigation, Formal analysis, Methodology, Software, Writing - review \& editing. Chiara Favaretto: Conceptualization, Investigation, Formal analysis, Methodology, Writing - review \& editing. Nicholas Metcalf: Data curation. Maurizio Corbetta: Conceptualization, Funding acquisition, Methodology, Supervision, Writing - review \& editing. Andrea Brovelli: Conceptualization, Funding acquisition, Investigation, Methodology, Supervision, Writing - review \& editing.

\section{Declaration of Competing Interest}

The authors declare that they have no known competing financial interests or personal relationships that could have appeared to influence the work reported in this paper.

\section{Acknowledgements}

MA, AB, CF and MC were supported by FLAG ERA II "Joint Transnational Call 2017“ - HBP - Basic and Applied Research 2, BrainsynchHit (ANR-17-HBPR-0001) during completion of this work. $\mathrm{AB}$ has received funding from the European Union's Horizon 2020 Framework Programme for Research and Innovation under the Specific Grant Agreement No. 945539 (Human Brain Project SGA3). The Authors thank Gustavo Deco and Reiner Goebel for helpful comments and suggestions during completion of this work, and Daniele Marinazzo for useful criticism and suggestions during revision of the manuscript.

\section{Data and code availability statement}

The full set of neuroimaging data (along with behavioral data) are available at http://cnda.wustl.edu/app/template/Login. The Granger Causality code used in this work is available at https://github.com/ micheleallegra/CovGC.

\section{References}

Aguirre, G.K., Zarahn, E., D'Esposito, M., 1998. The variability of human, BOLD hemodynamic responses. Neuroimage 8 (4), 360-369.

Angelini, L., De Tommaso, M., Marinazzo, D., Nitti, L., Pellicoro, M., Stramaglia, S., 2010. Redundant variables and Granger causality. Physical Review E 81 (3), 037201. Baldassarre, A., Ramsey, L., Hacker, C.L., Callejas, A., Astafiev, S.V., Metcalf, N.V., Zinn, K., Rengachary, J., Snyder, A.Z., Carter, A.R., Shulman, G.L., Corbetta, M., 2014. Large-scale changes in network interactions as a physiological signature of spatial neglect. Brain 137, 3267-3283. https://doi.org/10.1093/brain/awu297. 
Barnett, L., Barrett, A.B., Seth, A.K., 2009. Granger Causality and Transfer Entropy Are Equivalent for Gaussian Variables. Phys. Rev. Lett. 103, 238701 https://doi.org/ 10.1103/PhysRevLett.103.238701.

Barnett, L., Seth, A.K., 2014. The MVGC multivariate Granger causality toolbox: A new approach to Granger-causal inference. J. Neurosci. Methods 223, 50-68. https://doi. org/10.1016/j.jneumeth.2013.10.018.

Beirlant, J., Dudewicz, E.J., Györfi, L., Van der Meulen, E.C., 1997. Nonparametric entropy estimation: An overview. Int. J. Math. Stat. Sci. 6, 17-39.

Besserve, M., Lowe, S.C., Logothetis, N.K., Schölkopf, B., Panzeri, S., 2015. Shifts of gamma phase across primary visual cortical sites reflect dynamic stimulusmodulated information transfer. PLoS Biol. 13 (9), e1002257.

Bressler, S.L., Seth, A.K., 2011. Wiener-Granger causality: a well established methodology. Neuroimage 58 (2), 323-329.

Brovelli, A., Chicharro, D., Badier, J.-M., Wang, H., Jirsa, V., 2015. Characterization of Cortical Networks and Corticocortical Functional Connectivity Mediating Arbitrary Visuomotor Mapping. J. Neurosci. 35 (37), 12643-12658. https://doi.org/10.1523/ JNEUROSCI.4892-14.2015.

Brovelli, A., Ding, M., Ledberg, A., Chen, Y., Nakamura, R., Bressler, S.L., 2004. Beta oscillations in a large-scale sensorimotor cortical network: directional influences revealed by Granger causality. Proc. Natl. Acad. Sci. U.S.A 101 (26), 9849-9854.

Bullmore, E.d., Sporns, O., 2009. Complex brain networks: graph theoretical analysis of structural and functional systems. Nat. Rev. Neurosci. 10 (3), 186-198. https://doi org $/ 10.1038 /$ nrn2575.

Caballero-Gaudes, C., Reynolds, R.C., 2017. Methods for cleaning the BOLD fMRI signal. Neuroimage 154, 128-149.

Canella, C., Rocchi, F., Noei, S., Gutierrez-Barragan, D., Coletta, L., Galbusera, A., ... \& Gozzi, A. (2020). Cortical silencing results in paradoxical fMRI overconnectivity. bioRxiv.

Carter, A.R., Astafiev, S.V., Lang, C.E., Connor, L.T., Rengachary, J., Strube, M.J., Pope, D.L.W., Shulman, G.L., Corbetta, M., 2010. Resting interhemispheric functional magnetic resonance imaging connectivity predicts performance after stroke. Ann. Neurol. 67, 365-375. https://doi.org/10.1002/ana.21905.

Chicharro, D., Ledberg, A., 2012. Framework to study dynamic dependencies in networks of interacting processes. Phys. Rev. E 86, 041901. https://doi.org/10.1103/ PhysRevE.86.041901.

Corbetta, M., Kincade, M.J., Lewis, C., Snyder, A.Z., Sapir, A., 2005. Neural basis and recovery of spatial attention deficits in spatial neglect. Nature neuroscience 8 (11) 1603-1610. https://www.nature.com/articles/nn1574.

Corbetta, M., Shulman, G.L., 2011. Spatial neglect and attention networks. Annual review of neuroscience 34, 569-599. https://www.annualreviews.org/doi/pdf/10 .1146/annurev-neuro-061010-113731.

Corbetta, M., Ramsey, L., Callejas, A., Baldassarre, A., Hacker, C., Siegel, J., Astafiev, S., Rengachary, J., Zinn, K., Lang, C., Connor, L., Fucetola, R., Strube, M., Carter, A. Shulman, G., 2015. Common Behavioral Clusters and Subcortical Anatomy in Stroke. Neuron 85 (5), 927-941. https://doi.org/10.1016/j.neuron.2015.02.027.

Corbetta, M., Siegel, J.S., Shulman, G.L., 2018. On the low dimensionality of behavioral deficits and alterations of brain network connectivity after focal injury. Cortex, In Memory of Professor Glyn Humphreys 107, 229-237. https://doi.org/10.1016/j. cortex.2017.12.017.

Damoiseaux, J.S., Rombouts, S.A.R.B., Barkhof, F., Scheltens, P., Stam, C.J., Smith, S.M., Beckmann, C.F., 2006. Consistent resting-state networks across healthy subjects. Proc. Natl. Acad. Sci. 103 (37), 13848-13853. https://doi.org/10.1073/ pnas.0601417103.

Deco, G., Vidaurre, D., Kringelbach, M.L., 2021. Revisiting the Global Workspace orchestrating the hierarchical organization of the human brain. Nature Human Behaviour 1-15.

Ding, M., Chen, Y., Bressler, S.L., 2006. 17 Granger causality: basic theory and application to neuroscience. Handbook of time series analysis: recent theoretical developments and applications, 437.

Eldaief, M.C., McMains, S., Hutchison, R.M., Halko, M.A., Pascual-Leone, A., 2017. Reconfiguration of Intrinsic Functional Coupling Patterns Following Circumscribed Network Lesions. Cereb. Cortex 27, 2894-2910. https://doi.org/10.1093/cercor/ bhw139.

Erdoğan, S.B., Tong, Y., Hocke, L.M., Lindsey, K.P., deB Frederick, B., 2016. Correcting for blood arrival time in global mean regression enhances functional connectivity analysis of resting state fMRI-BOLD signals. Frontiers in human neuroscience 10, 311.

Faes, L., Stramaglia, S., Marinazzo, D., 2017. On the interpretability and computational reliability of frequency-domain Granger causality. F1000Research 6. https://doi. org/10.12688/f1000research.12694.1.

Fischl, B. (2012). FreeSurfer. Neuroimage. 62(2). 774-781.

Fischl, B., Sereno, M.I., Tootell, R.B., Dale, A.M., 1999a. High-resolution intersubject averaging and a coordinate system for the cortical surface. Hum. Brain Mapp. 8 (4), 272-284.

Fischl, B., Sereno, M.I., Dale, A.M., 1999b. Cortical surface-based analysis: II: inflation, flattening, and a surface-based coordinate system. Neuroimage 9 (2), 195-207.

Fischl, B., Salat, D.H., Busa, E., Albert, M., Dieterich, M., Haselgrove, C., van der Kouwe, A., Killiany, R., Kennedy, D., Klaveness, S., Montillo, A., Makris, N., Rosen, B., Dale, A.M., 2002. Whole brain segmentation: automated labeling of neuroanatomical structures in the human brain. Neuron 33 (3), 341-355.

Fox, M.D., Snyder, A.Z., Vincent, J.L., Corbetta, M., Essen, D.C.V., Raichle, M.E., 2005. The human brain is intrinsically organized into dynamic, anticorrelated functional networks. Proc. Natl. Acad. Sci. 102, 9673-9678. https://doi.org/10.1073/ pnas. 0504136102.
Friston, Karl, Moran, Rosalyn, Seth, Anil K, 2013. Analysing connectivity with Granger causality and dynamic causal modelling. Curr. Opin. Neurobiol. Macrocircuits 23 (2), 172-178. https://doi.org/10.1016/j.conb.2012.11.010.

Geweke, John, 1982. Measurement of Linear Dependence and Feedback between Multiple Time Series. J. Am. Stat. Assoc. 77 (378), 304-313. https://doi.org/ 10.1080/01621459.1982.10477803.

Glasser, M.F., Sotiropoulos, S.N., Wilson, J.A., Coalson, T.S., Fischl, B., Andersson, J.L., Xu, J., Jbabdi, S., Webster, M., Polimeni, J.R., Van Essen, D.C., Jenkinson, M., WuMinn HCP Consortium, 2013. The minimal preprocessing pipelines for the Human Connectome Project. Neuroimage 80, 105-124.

Golestani, Ali-Mohammad, Tymchuk, Sarah, Demchuk, Andrew, Goodyear, Bradley G., 2013. Longitudinal Evaluation of Resting-State fMRI After Acute Stroke With Hemiparesis. Neurorehabil. Neural Repair 27 (2), 153-163. https://doi.org/ 10.1177/1545968312457827.

Gordon, Evan M., Laumann, Timothy O., Adeyemo, Babatunde, Huckins, Jeremy F., Kelley, William M., Petersen, Steven E., 2016. Generation and Evaluation of a Cortical Area Parcellation from Resting-State Correlations. Cereb. Cortex 26 (1), 288-303. https://doi.org/10.1093/cercor/bhu239.

Gotts, Stephen J., Jo, Hang Joon, Wallace, Gregory L., Saad, Ziad S., Cox, Robert W., Martin, Alex, 2013. Two distinct forms of functional lateralization in the human brain. Proc. Natl. Acad. Sci. 110 (36), E3435-E3444. https://doi.org/10.1073/ pnas. 1302581110.

Granger, C.W.J., 1969. Investigating Causal Relations by Econometric Models and Crossspectral Methods. Econometrica 37, 424-438. https://doi.org/10.2307/1912791.

Gratton, C., Nomura, E.M., Pérez, F., D’Esposito, M., 2012. Focal Brain Lesions to Critical Locations Cause Widespread Disruption of the Modular Organization of the Brain. J. Cogn. Neurosci. 24, 1275-1285. https://doi.org/10.1162/jocn_a_00222.

Grefkes, Christian, Fink, Gereon R, 2014. Connectivity-based approaches in stroke and recovery of function. Lancet Neurol. 13 (2), 206-216. https://doi.org/10.1016/ S1474-4422(13)70264-3.

Griffis, Joseph C., Metcalf, Nicholas V., Corbetta, Maurizio, Shulman, Gordon L., 2020. Damage to the shortest structural paths between brain regions is associated with disruptions of resting-state functional connectivity after stroke. NeuroImage 210, 116589. https://doi.org/10.1016/j.neuroimage.2020.116589.

Griffis, Joseph C., Metcalf, Nicholas V., Corbetta, Maurizio, Shulman, Gordon L., 2019. Structural Disconnections Explain Brain Network Dysfunction after Stroke. Cell Rep. 28 (10), 2527-2540.e9. https://doi.org/10.1016/j.celrep.2019.07.100.

Hahamy, Avital, Calhoun, Vince, Pearlson, Godfrey, Harel, Michal, Stern, Nachum, Attar, Fanny, Malach, Rafael, Salomon, Roy, 2014. Save the Global: Global Signal Connectivity as a Tool for Studying Clinical Populations with Functional Magnetic Resonance Imaging. Brain Connect. 4 (6), 395-403. https://doi.org/10.1089/ brain. 2014.0244.

He, Biyu J., Snyder, Abraham Z., Vincent, Justin L., Epstein, Adrian, Shulman, Gordon L., Corbetta, Maurizio, 2007. Breakdown of Functional Connectivity in Frontoparietal Networks Underlies Behavioral Deficits in Spatial Neglect. Neuron 53 (6), 905-918. https://doi.org/10.1016/j.neuron.2007.02.013.

Hlinka, Jaroslav, Paluš, Milan, Vejmelka, Martin, Mantini, Dante, Corbetta, Maurizio, 2011. Functional connectivity in resting-state fMRI: Is linear correlation sufficient? NeuroImage 54 (3), 2218-2225. https://doi.org/10.1016/j. neuroimage.2010.08.042

Ince, Robin A.A., Giordano, Bruno L., Kayser, Christoph, Rousselet, Guillaume A., Gross, Joachim, Schyns, Philippe G., 2017. A statistical framework for neuroimaging data analysis based on mutual information estimated via a gaussian copula. Hum. Brain Mapp. 38 (3), 1541-1573. https://doi.org/10.1002/hbm.23471.

Iturria-Medina, Y., Pérez Fernández, A., Morris, D.M., Canales-Rodríguez, E.J., Haroon, H.A., García Pentón, L., Augath, M., Galán García, L., Logothetis, N., Parker, G.J.M., Melie-García, L., 2011. Brain Hemispheric Structural Efficiency and Interconnectivity Rightward Asymmetry in Human and Nonhuman Primates. Cereb. Cortex 21, 56-67. https://doi.org/10.1093/cercor/bhq058.

Karolis, V.R., Corbetta, M., Thiebaut de Schotten, M., 2019. The architecture of functional lateralisation and its relationship to callosal connectivity in the human brain. Nat. Commun. 10, 1417. https://doi.org/10.1038/s41467-019-09344-1.

Liu, T.T., Nalci, A., Falahpour, M., 2017. The global signal in fMRI: Nuisance or Information? NeuroImage 150, 213-229. https://doi.org/10.1016/j. neuroimage.2017.02.036.

Marcus, D.S., Harms, M.P., Snyder, A.Z., Jenkinson, M., Wilson, J.A., Glasser, M.F., Barch, D.M., Archie, K.A., Burgess, G.C., Ramaratnam, M., Hodge, M., Horton, W., Herrick, R., Olsen, T., McKay, M., House, M., Hileman, M., Reid, E., Harwell, J., Coalson, T., Schindler, J., Elam, J.S., Curtiss, S.W., Van Essen, D.C., 2013. WU-Minn HCP Consortium, Human Connectome Project informatics: quality control, database services, and data visualization. Neuroimage 80, 202-219.

Marko, H., 1973. The Bidirectional Communication Theory - A Generalization of Information Theory. IEEE Trans. Commun. 21 (12), 1345-1351. https://doi.org/ 10.1109/TCOM.1973.1091610.

Massey, J., 1990. Causality, feedback and directed information. In Proc. Int. Symp. Inf. Theory Applic. (ISITA-90) 303-305.

New, A.B., Robin, D.A., Parkinson, A.L., Duffy, J.R., McNeil, M.R., Piguet, O., Hornberger, M., Price, C.J., Eickhoff, S.B., Ballard, K.J., 2015. Altered resting-state network connectivity in stroke patients with and without apraxia of speech. NeuroImage Clin. 8, 429-439. https://doi.org/10.1016/j.nicl.2015.03.013.

Park, Chang-hyun, Chang, Won Hyuk, Ohn, Suk Hoon, Kim, Sung Tae, Bang, Oh Young, Pascual-Leone, Alvaro, Kim, Yun-Hee, 2011. Longitudinal Changes of Resting-State Functional Connectivity During Motor Recovery After Stroke. Stroke 42 (5), 1357-1362. https://doi.org/10.1161/STROKEAHA.110.596155.

Pino, G.D., Pellegrino, G., Assenza, G., Capone, F., Ferreri, F., Formica, D., Ranieri, F., Tombini, M., Ziemann, U., Rothwell, J.C., Di Lazzaro, V., 2014. Modulation of brain 
plasticity in stroke: a novel model for neurorehabilitation. Nat. Rev. Neurol. 10 (10), 597-608. https://doi.org/10.1038/nrneurol.2014.162.

Ramsey, Lenny E., Siegel, Joshua S., Baldassarre, Antonello, Metcalf, Nicholas V., Zinn, Kristina, Shulman, Gordon L., Corbetta, Maurizio, 2016. Normalization of network connectivity in hemispatial neglect recovery. Ann. Neurol. 80 (1), 127-141. https://doi.org/10.1002/ana.24690.

Rehme, A.K., Grefkes, C., 2013. Cerebral network disorders after stroke: evidence from imaging-based connectivity analyses of active and resting brain states in humans. J. Physiol. 591, 17-31. https://doi.org/10.1113/jphysiol.2012.243469.

Rissanen, J., Wax, M., 1987. Measures of mutual and causal dependence between two time series (Corresp.). IEEE Trans. Inf. Theory 33 (4), 598-601. https://doi.org/ 10.1109/TIT.1987.1057325.

Roebroeck, Alard, Formisano, Elia, Goebel, Rainer, 2011. The identification of interacting networks in the brain using fMRI: Model selection, causality and deconvolution. NeuroImage 58 (2), 296-302. https://doi.org/10.1016/j. neuroimage.2009.09.036.

Roebroeck, Alard, Formisano, Elia, Goebel, Rainer, 2005. Mapping directed influence over the brain using Granger causality and fMRI. NeuroImage 25 (1), 230-242. https://doi.org/10.1016/j.neuroimage.2004.11.017.

Saad, Ziad S., Gotts, Stephen J., Murphy, Kevin, Chen, Gang, Jo, Hang Joon, Martin, Alex, Cox, Robert W., 2012. Trouble at Rest: How Correlation Patterns and Group Differences Become Distorted After Global Signal Regression. Brain Connect. 2 (1), 25-32. https://doi.org/10.1089/brain.2012.0080.

Schmahmann, J.D., Schmahmann, J., Pandya, D., 2009. Fiber Pathways of the Brain. Oxford University Press, USA.

Scholvinck, M.L., Maier, A., Ye, F.Q., Duyn, J.H., Leopold, D.A., 2010. Neural basis of global resting-state fMRI activity. Proc. Natl. Acad. Sci. 107 (22), 10238-10243. https://doi.org/10.1073/pnas.0913110107.

Schreiber, Thomas, 2000. Measuring Information Transfer. Phys. Rev. Lett. 85 (2), 461-464. https://doi.org/10.1103/PhysRevLett.85.461.

Seth, A.K., Barrett, A.B., Barnett, L., 2015. Granger causality analysis in neuroscience and neuroimaging. J. Neurosci. 35 (8), 3293-3297.

Siegel, Joshua Sarfaty, Ramsey, Lenny E., Snyder, Abraham Z., Metcalf, Nicholas V., Chacko, Ravi V., Weinberger, Kilian, Baldassarre, Antonello, Hacker, Carl D., Shulman, Gordon L., Corbetta, Maurizio, 2016a. Disruptions of network connectivity predict impairment in multiple behavioral domains after stroke. Proc. Natl. Acad. Sci. 113 (30), E4367-E4376. https://doi.org/10.1073/pnas.1521083113.

Siegel, Joshua S, Snyder, Abraham Z, Ramsey, Lenny, Shulman, Gordon L, Corbetta, Maurizio, 2016b. The effects of hemodynamic lag on functional connectivity and behavior after stroke. J. Cereb. Blood Flow Metab. 36 (12), 2162-2176. https://doi.org/10.1177/0271678X15614846.

Stramaglia, Sebastiano, et al., 2016. Synergetic and redundant information flow detected by unnormalized Granger causality: Application to resting state fMRI. IEEE Transactions on Biomedical Engineering 63 (12), 2518-2524.

Stramaglia, Sebastiano, M Cortes, Jesus, Marinazzo, Daniele, 2014. Synergy and redundancy in the Granger causal analysis of dynamical networks. New Journal of Physics 16 (10), 105003. https://doi.org/10.1088/1367-2630/16/10/105003.

Tang, Chaozheng, Zhao, Zhiyong, Chen, Chuang, Zheng, Xiaohui, Sun, Fenfen, Zhang, Xiaoli, Tian, Jing, Fan, Mingxia, Wu, Yi, Jia, Jie, Duce, James A., 2016. Decreased Functional Connectivity of Homotopic Brain Regions in Chronic Stroke Patients: A Resting State fMRI Study. PLoS ONE 11 (4), e0152875. https://doi.org/ 10.1371/journal.pone.0152875.

Tang, Wei, Bressler, Steven L., Sylvester, Chad M., Shulman, Gordon L., Corbetta, Maurizio, Sporns, Olaf, 2012. Measuring Granger causality between cortical regions from voxelwise fMRI BOLD signals with LASSO. PLoS Comput Biol 8 (5), e1002513. https://doi.org/10.1371/journal.pcbi.1002513.

Treves, Alessandro, Panzeri, Stefano, 1995. The Upward Bias in Measures of Information Derived from Limited Data Samples. Neural Comput. 7 (2), 399-407. https://doi. org/10.1162/neco.1995.7.2.399.

Van Essen, David C., Smith, Stephen M., Barch, Deanna M., Behrens, Timothy E.J., Yacoub, Essa, Ugurbil, Kamil, 2013. The WU-Minn human connectome project: an overview. Neuroimage 80, 62-79.

Vicente, R., Wibral, M., Lindner, M., Pipa, G., 2011. Transfer entropy-a model-free measure of effective connectivity for the neurosciences. J. Comput. Neurosci. 30 (1), 45-67.

Wang, Xin, Seguin, Caio, Zalesky, Andrew, Wong, Wan-wa, Chu, Winnie Chiu-wing, Tong, Raymond Kai-yu, 2019. Synchronization lag in post stroke: relation to motor function and structural connectivity. Network Neuroscience 3 (4), 1121-1140.

Wu, Guo-Rong, Liao, Wei, Stramaglia, Sebastiano, Ding, Ju-Rong, Chen, Huafu, Marinazzo, Daniele, 2013. A blind deconvolution approach to recover effective connectivity brain networks from resting state fMRI data. Medical image analysis 17 (3), 365-374.

Yang, G.J., Murray, J.D., Repovs, G., Cole, M.W., Savic, A., Glasser, M.F., Pittenger, C., Krystal, J.H., Wang, X.-J., Pearlson, G.D., Glahn, D.C., Anticevic, A., 2014. Altered global brain signal in schizophrenia. Proc. Natl. Acad. Sci. 111 (20), 7438-7443. https://doi.org/10.1073/pnas.1405289111. 